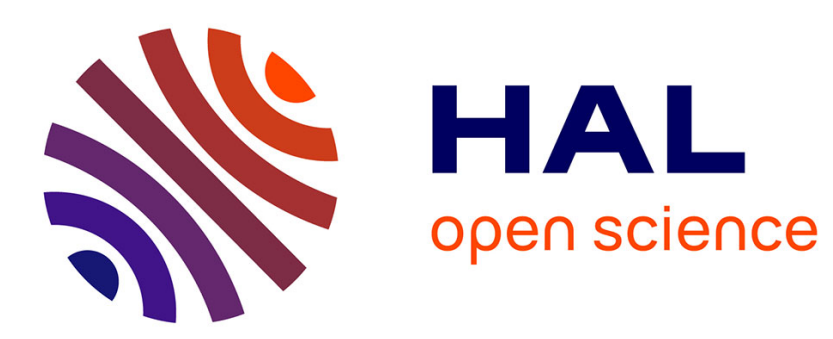

\title{
FFT based numerical homogenization method for porous conductive materials
}

\author{
Quy-Dong To, Guy Bonnet
}

\section{To cite this version:}

Quy-Dong To, Guy Bonnet. FFT based numerical homogenization method for porous conductive materials. Computer Methods in Applied Mechanics and Engineering, 2020, 368, pp.113160. 10.1016/j.cma.2020.113160 . hal-02794345

\section{HAL Id: hal-02794345 \\ https://hal.science/hal-02794345}

Submitted on 5 Jun 2020

HAL is a multi-disciplinary open access archive for the deposit and dissemination of scientific research documents, whether they are published or not. The documents may come from teaching and research institutions in France or abroad, or from public or private research centers.
L'archive ouverte pluridisciplinaire HAL, est destinée au dépôt et à la diffusion de documents scientifiques de niveau recherche, publiés ou non, émanant des établissements d'enseignement et de recherche français ou étrangers, des laboratoires publics ou privés. 


\title{
FFT based numerical homogenization method for porous conductive materials
}

\author{
Quy-Dong To*, Guy Bonnet
}

Université Paris-Es, Laboratoire Modelisation et Simalation Multi Echelle, MSME UMR 8208 CNRS, 5 Boulevard Descartes, 77454

Marne-la-Vallée Cedex 2, France

\begin{abstract}
The Fourier series method is used to solve the periodic homogenization problem for conductive materials containing voids. The problems involving voids are special cases of infinite contrast whose full field solution is not unique, causing convergence issues when iteration schemes are used. In this paper, we reformulate the problem based on the temperature field in the skeleton and derive an equation where the temperature field is connected to values on the pore boundary. Iteration schemes based on the new equation show that the convergence is fast, yielding good results both in terms of local fields and effective conductive properties.
\end{abstract}

Keywords: Fast Fourier transform; Numerical homogenization method; Porous conductive media; Iteration scheme 
homogeneous medium, whose Fourier components are known explicitly. Other advantages are the use of a reduced memory storage for computation and the ease of handling tomography images displayed on a regular grid. On the other hand, the methods are also subject to several limitations including the convergence issues at extreme values of contrast ratio, as seen thereafter.

In this paper, we address the case of porous materials where the contrast of physical parameters is infinite. The present paper is devoted to conduction problems. However, numerous results of the literature were obtained either for conduction or for elasticity. These problems are fundamentally of the same kind (elliptic second order operators). The following discussion will refer to both types of problems. The first formulation of the FFT method [4] was based in elasticity on the formulation of an integral equation of Lippmann-Schwinger-Dyson type (as in [3] for conduction) whose kernel is the Green tensor in Fourier space computed from the properties of a reference medium. The Green tensor acts on the local polarization computed from the local elastic properties and the ones of the reference medium. In the first version of the method, the solution is obtained by building a Neumann series to solve this integral equation. The difficulty to deal with high contrasts when using such a formulation was early recognized. In the case of the iterative scheme based on Neumann series, compared with 3 other schemes, Michel et al. [11] have shown that the eigenvalues of the iterative operator can be bounded with convenient values of the physical parameters of the "reference medium" used in the expression of the Lippmann-Schwinger equation. If the upper bound of these eigenvalues is strictly inferior to 1, the convergence is ensured. For very small or very high contrasts, the eigenvalues are nearer to 1 , reducing the speed of convergence.

In the case of infinite contrast, such a bounding is no more possible: the eigenvalues of the continuous iterative operator have eigenvalues very near to 1 and its discrete version has eigenvalues whose upper bound increases toward 1 with the discretization size. Several methods have been provided in order to improve the convergence of the iterative process at high contrast $[6,7,9]$. However, if one requires for the local field to be physically consistent, i.e. complying with the expression of the conservation law in discrete Fourier space: (equilibrium in the case of elasticity, or conservation of energy in the case of conduction, denoted thereafter CLF, Conservation Law in discrete Fourier space), it was shown by Moulinec and Silva [12] that the convergence does not occur with the mostly used schemes: basic scheme, accelerated schemes, lagrangian scheme, Eyre-Milton scheme. It is noticeable that the use of CLF leads to satisfying solutions in the case of finite contrast. However, its use in the most general case was considered as unreliable by Schneider et al. [13] who stated that "from the point of view of functional analysis, the divergence measured by this criterion does not imply the divergence of the solution". Since the CLF criterion is very demanding and provides satisfying solutions in the case of finite contrast, it is important to consider this aspect in the present discussion.

In the same time, the numerical analysis of the solution procedure led to significant improvements. The estimation of error produced by the iterative process for high contrasts has been studied [14,15], the solution of the cell problem by the original method has been proved to be equivalent to a Galerkin discretization $[16,17]$. An important step was to combine the FFT method with the conjugate gradient method $[10,18]$ and with other efficient Newton-Krylov solvers [19-21]. These methods improve significantly the convergence rate. However, it has been shown that the conjugate gradient method does not converge at infinite contrast $[18,22]$ when using the CLF criterion. This is not the case for less demanding criteria, as for example in [10] which found a solution described as comparing well with a finite element one. The Barzilai-Borwein basic scheme has been claimed to be able to provide convergence even in the case of porous media [19]. However, the example provided in this paper deals with a very small porosity (around $1 \%$ ) in the case of a complex microstructure. From a fundamental point of view, the convergence of the Barzilai-Borwein scheme is ensured only with coercive operators, which is not the case for porous materials. Further work with a comparison of the solution obtained from Barzilai-Borwein scheme with exact solutions would be necessary to conclude on this point.

Up to now, we have considered in this discussion the extensions of the basic scheme using the Green tensor in Fourier space introduced in the original paper on the method, coming from the basic conservation equation. However, several works were using a different path: they built a Lippmann-Schwinger equation using an "alternative Green tensor" based on a different formulation [23-27], while being as valid as the usual Green tensor. It seems that the convergence of the iterative process is improved, leading to convergence also in the case of porous media. However, in this case, the expression of the conservation law is different from the one used for the basic scheme. As a consequence, the convergence criterion is different from the one used in the methods based on the "classical" Green tensor: the comparison may be biased by this fundamental difference. In this study, we shall keep the classical Green tensor. 
In the literature on FFT methods, a puzzling point has been noticed several times: the general results on the convergence in the case of voids show that the convergence criteria are not met [11]. However, it has been often claimed that the convergence of the effective tensor can be achieved. An enlightening answer to this point has been given in a recent paper [28]: the convergence of effective properties can be met even in the case of cells containing voids if some additional information on the cell is available, even if the convergence of the local field is not achieved. The authors studied the case of conduction in the case of a squared inclusion whose analytical solution is known, both for the effective conductivity and the local field. Scrutinizing the expansion of the effective conductivity as a series of terms containing the contrast of permeabilities, they have shown that a significant set of coefficients of the expansion are estimated correctly by the numerical solutions, even in the case of void containing materials. They have also shown that the convergence of different schemes, including the case of Willot [23] using the "alternative Green tensor", provide the effective conductivity with a relative error around $10^{-4}$, even for voids. However, the convergence criterion on the conservation equation was not achieved in the case of the conventional Green tensor in the case of voids. Surprisingly, the convergence using the conservation equation was achieved using the "alternative Green tensor", but does not lead to a better convergence of the effective conductivity.

At the end of our discussion of the literature, one can see that numerous FFT methods have been introduced: they differ by the kind of Green tensor, the criterion of convergence, the numerical solver, etc. They can also differ by the kind of reconstruction of the solution from the values obtained at discretized points. This will not be considered thereafter.

From the previous survey of the literature, the convergence of FFT methods in the case of materials containing voids is still challenging. In the following, we shall address the case of conduction in porous materials. To circumvent the difficulty when dealing with infinite contrasts, one can remark that the iterative operator is fundamentally affected in the case of voids: the elasticity tensor within the cell is no more coercive within all the cells. As a consequence, the full field solution is no longer unique: the solution is obtained up to the addition of any field null outside the inclusions. Comparing the solution of that problem with FEM procedures, one can see that the problem would not be solved using finite elements with a discretization that would contain the voids. On the contrary, excluding the void from the finite element mesh leads to a coercive elasticity tensor and a unique solution. The main idea of the present work to circumvent the problem related to voids is to take as a main variable the field outside the voids.

To formulate the problem, we start from a formulation based on temperature. To ensure the uniqueness of the solution, we modify the formulation in order to solve the temperature field in the skeleton only. In the newly derived equation, the temperature field is a function of the distribution of temperature on the pore boundary. The iteration scheme is then formulated in Fourier space using form factors for elliptical or polygonal shapes [29-32] of the pore. The local solution fields and the effective conductivity are finally compared with those coming from the Finite Element Method and from closed form solutions. The details will be presented in the following sections.

\section{Formulation and resolution method}

\subsection{Classical integral equation for temperature gradient}

Before proceeding, let us introduce the notations used in the paper. The bold characters $A, \boldsymbol{u}$ represent vectors or tensors and the normal characters $\varphi$ are used for scalars. We shall denote the Fourier transform of a $V$-periodic function $\boldsymbol{u}(\boldsymbol{x})$ of cartesian coordinates $\boldsymbol{x}\left(x_{1}, x_{2}, x_{3}\right)$ as $\boldsymbol{u}(\boldsymbol{\xi})$

$$
\boldsymbol{u}(\xi)=\frac{1}{V} \int_{V} \boldsymbol{u}(\boldsymbol{x}) e^{-i \xi x} d \boldsymbol{x}, \quad i=\sqrt{-1}
$$

with $\xi\left(\xi_{1}, \xi_{2}, \xi_{3}\right)$ being the wave vector

$$
\xi_{k}=2 \pi n_{k} / L_{k}, \quad n_{k}=0, \pm 1, \pm 2, \ldots, \pm \infty, \quad k=1,2,3
$$

and $L_{1}, L_{2}, L_{3}$ being the dimensions of the period $V$ along direction $x_{1}, x_{2}, x_{3}$. The quantities standing next to each other imply normal or contracted product, $x$ for vector product and $*$ for convolution product. We note that in physical space, it can be expressed as

$$
A(\boldsymbol{x}) * \boldsymbol{u}(\boldsymbol{x})=\sum_{\xi} \boldsymbol{A}(\xi) \boldsymbol{u}(\xi) e^{i \xi x}
$$


and in Fourier space the convolution is discrete,

$$
A(\xi) * u(\xi)=\sum_{\xi^{\prime}} A\left(\xi-\xi^{\prime}\right) \boldsymbol{u}\left(\xi^{\prime}\right)
$$

Let us consider a heterogeneous media where heat conductivity $k(x)$ is a periodic function of coordinates $x\left(x_{1}, x_{2}, x_{3}\right)$ with period $V$, a rectangular box of dimension $L_{1} \times L_{2} \times L_{3}$. To find the effective properties of the material, the usual method is to solve the conduction problem in a unit cell $V$ with periodicity boundary conditions. The domain is free of heat source and the average temperature gradient $\boldsymbol{E}$ or average flux $\boldsymbol{J}$ is prescribed. In the former case ( $\boldsymbol{E}$ prescribed), a current method is to solve the following classical integral equation valid for any constant conductivity $k_{0}[3,8,33]$

$$
\boldsymbol{e}(\boldsymbol{x})=\boldsymbol{E}-\boldsymbol{P} *\left(\delta k / k_{0}\right) \boldsymbol{e}(\boldsymbol{x}), \quad \delta k(\boldsymbol{x})=k(\boldsymbol{x})-k_{0}
$$

where $e$ is the (minus) gradient of temperature $T$ or $e=-\nabla T$ and $*$ the convolution operator. In Fourier space, $\boldsymbol{P}$ is a tensor function of wavevector $\boldsymbol{\xi}\left(\xi_{1}, \xi_{2}, \xi_{3}\right)$ defined as follows

$$
P(\xi)=\bar{\xi} \otimes \bar{\xi} \quad \forall \xi \neq 0, \quad P(\xi=0)=0 .
$$

with $\bar{\xi}=\xi / \xi$ and $\xi=|\xi|$.

\subsection{Integral equation for temperature}

We reformulate now the above equation in terms of temperature. We remark that $\boldsymbol{e}$ has a non zero average $\boldsymbol{E}$ and cannot be derived from a periodic temperature field $T(\boldsymbol{x})$. Instead, we use a periodic function $\theta(\boldsymbol{x})$ defined as

$$
T(x)=-E x-\theta(x), \quad \text { or } \quad \nabla \theta(x)=e(x)-E
$$

In this case, $\theta$ is the fluctuation of temperature. However, $T$ will be no more used and $\theta$ will be named "temperature" in the following. In Fourier space, $\theta$ is related to $e$ via the relation

$$
e(\xi)=(\nabla \theta)(\xi)=i \xi \theta(\xi) \quad \forall \xi \neq 0
$$

We can obtain the integral equation for temperature

$$
\theta=\theta+R *\left(\delta k / k_{0}\right)(\nabla \theta+E)
$$

with $\theta$ the average temperature $\theta$, i.e. $\theta=\langle\theta\rangle$ and the newly derived operator $\boldsymbol{R}[21,34]$ given by:

$$
\boldsymbol{R}(\xi)=\frac{i \xi}{\xi^{2}}=\frac{i \bar{\xi}}{\xi}, \quad \boldsymbol{R}(\xi=0)=0
$$

The above integral equation can be solved by using an iteration scheme with $E$ as prescribed action. The constant $\Theta$ can be set arbitrarily, for example $\Theta=0$, without affecting $e$ and the obtained effective conductivity.

There are some properties of $\boldsymbol{R}$ that we need to mention for later use. First, from the definition of $\boldsymbol{R}$, we have for any $\theta$ :

$$
\theta(\boldsymbol{\xi})=-\boldsymbol{R}(\boldsymbol{\xi})(i \xi \theta(\boldsymbol{\xi})) \quad \forall \boldsymbol{\xi} \neq 0, \quad \text { or } \quad \theta(\boldsymbol{x})-\langle\theta\rangle=-\boldsymbol{R} * \nabla \theta(\boldsymbol{x})
$$

This is due to the fact that we have, from $(10), \boldsymbol{R}(\boldsymbol{\xi}) i \xi=-\boldsymbol{I} \forall \boldsymbol{\xi} \neq 0$ where $\boldsymbol{I}$ is the second order identity tensor.

Second, for all divergence free field $j(x)$, we have $i \xi j(\xi)=0$, or equivalently

$$
\boldsymbol{R}(\xi) \boldsymbol{j}(\xi)=0 \quad \forall \xi, \quad \text { or } \quad \boldsymbol{R} * \boldsymbol{j}(\boldsymbol{x})=0
$$

Another remark is that the solution $\theta(x)$ exists uniquely if $k(x)>0 \forall x \in V$ or $k(x)<0 \forall x \in V$. In the case of a porous material, i.e., $\exists x$ so that $k(x)=0$, the solution is not unique. While the temperature in the skeleton may be well defined and unique, the temperature in the void can be any continuation of the latter from the boundary. The consequence is that there exists a non trivial solution $\theta(x) \neq 0$ at zero loading condition $E=0$, or

$$
\exists \theta(\boldsymbol{x}) \neq 0, \quad \theta=\boldsymbol{R} *\left(\delta k / k_{0}\right)(\nabla \theta)
$$

The eigen equation associated with the operator $\boldsymbol{R} *\left(\delta k / k_{0}\right)(\nabla)$ present in (9) reads:

$$
\lambda \theta=R *\left(\delta k / k_{0}\right)(\nabla \theta)
$$


We find therefore that there is an eigenvalue $\lambda=1$. As a result, using iterative schemes to solve $\theta(x)$ in $(9)$ does not ensure the convergence of the iterative process. This result has been found when studying all the previously mentioned iterative processes.

To deal with the above issues, the main idea developed in the following is to avoid obtaining the full field $\theta(\boldsymbol{x})$ and look instead for the part of this field related only to the skeleton.

\subsection{Consideration of a porous material}

Let us assume that the cell $V$ is composed of voids occupying volume $\Omega_{v}$ with a characteristic function $\chi_{v}(x)$ and $k(x)=0$, the regular zone is homogeneous, occupying volume $\Omega_{r}$ with a characteristic function $\chi_{r}(x)$ and a finite positive conductivity $k(\boldsymbol{x})=k_{r}$ (see Fig. 1). Specifically, the conductivity distribution of the whole material reads

$$
k(x)=k_{r} \chi_{r}(x) \quad \forall x, \quad 0<k_{r}<\infty,
$$

and

$$
\begin{array}{ll}
\chi_{v}(x)=1 \text { in } \Omega_{v}, & \chi_{v}(x)=0 \text { in } \Omega_{r} \\
\chi_{r}(x)=1 \text { in } \Omega_{r}, & \chi_{r}(x)=0 \text { in } \Omega_{v}
\end{array}
$$

We continue to use the notations $\theta, e$ and $j$ for the temperature, gradient and flux field of the whole domain $V$ while denoting the same quantities in the sub-domains $\left(\theta_{v}, \boldsymbol{e}_{v}\right)$ for voids, $\left(\theta_{r}, \boldsymbol{e}_{r}\right)$ for regular domain. They are defined via the mathematical expressions

$$
\theta_{\alpha}(x)=\chi_{\alpha}(x) \theta(x) \quad \boldsymbol{e}_{\alpha}(x)=\chi_{\alpha}(x) e(x), \quad \alpha=r, v
$$

We know that if the problem is well posed, the solution $\theta_{r}$ in $\Omega_{r}$ exists uniquely while $\theta_{v}$ in the void $\Omega_{v}$ is not unique. The field $\theta_{v}$ can be any continuation function of $\theta_{r}$ into the void. This may cause issues when using the numerical methods based on the governing equation. To overcome those difficulties, we shall reformulate the problem based on temperature variable $\theta_{r}$ in $\Omega_{r}$.

First our integral equation (9) can be decomposed into the following form

$$
\theta=\theta+R * \frac{k_{r}-k_{0}}{k_{0}} \chi_{r}(\nabla \theta+E)-R * \chi_{v}(\nabla \theta+E)
$$

Next, we examine the term $\boldsymbol{e}_{\alpha}=\chi_{\alpha}(\nabla \theta+E)$ in Fourier space, which yields the results

$$
\begin{aligned}
& e_{\alpha}(\xi)=\chi_{\alpha}(E+\nabla \theta)(\xi)=E \chi_{\alpha}(\xi)+\frac{1}{V} \int_{\Omega_{\alpha}} \nabla \theta(x) e^{-i \xi \cdot x} d x \\
& =E \chi_{\alpha}(\xi)+\frac{1}{V} \int_{\Gamma_{\alpha}} \theta(\boldsymbol{x}) \boldsymbol{n}(\boldsymbol{x}) e^{-i \xi \cdot x} d s+i \xi \frac{1}{V} \int_{\Omega_{\alpha}} \theta(\boldsymbol{x}) e^{-i \xi \cdot x} d \boldsymbol{x} \\
& =E \chi_{\alpha}(\xi)+\left[(\boldsymbol{n} \delta)_{\Gamma_{\alpha}} \theta\right](\xi)+i \xi \theta_{\alpha}(\xi)
\end{aligned}
$$

with $n$ being the outward normal vector of the boundary $\Gamma_{\alpha}$ of $\Omega_{\alpha}$ and

$$
\left[(\boldsymbol{n} \delta)_{\Gamma_{\alpha}} \theta\right](\xi)=\frac{1}{V} \int_{\Gamma_{\alpha}} \theta(\boldsymbol{x}) \boldsymbol{n}(\boldsymbol{x}) e^{-i \xi \cdot x} d s,
$$

Note that we have integrated by parts and transformed the volume integral $\Omega_{\alpha}$ into a surface integral on $\Gamma_{\alpha}$ using the Gauss theorem. In this relation, $(\boldsymbol{n} \delta)_{\Gamma_{\alpha}} \theta$ is the product of the continuous function $\theta(\boldsymbol{x})$ and of the distribution $(\boldsymbol{n} \delta)_{\Gamma_{\alpha}}(\boldsymbol{x})$ associated with interface $\Gamma_{\alpha}$. The latter, viewed as surface delta distribution $\delta$ multiplied with the local normal vector $\boldsymbol{n}$, can be defined as

$$
\frac{1}{V} \int_{V}(n \delta)_{\Gamma_{\alpha}}(x) \varphi(x) d x=\frac{1}{V} \int_{\Gamma_{\alpha}} \boldsymbol{n}(x) \varphi(x) d s \quad \forall \varphi(x)
$$

for smooth test function $\varphi(\boldsymbol{x})$. Its Fourier transform admits a simple form

$$
(\boldsymbol{n} \delta)_{\Gamma_{\alpha}}(\boldsymbol{\xi})=\frac{1}{V} \int_{\Gamma_{\alpha}} \boldsymbol{n}(\boldsymbol{x}) e^{-i \xi x} d s=-i \xi \chi_{\alpha}(\xi)
$$



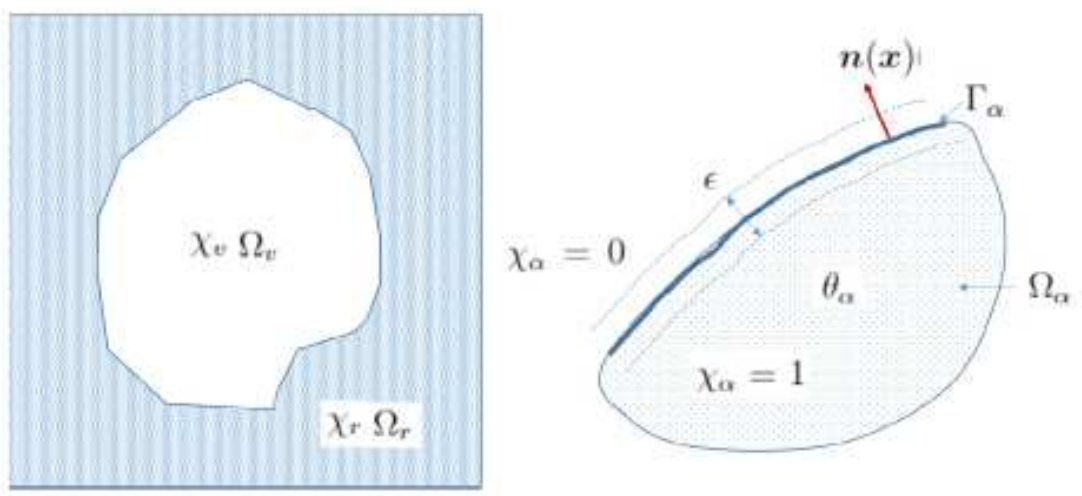

Fig. 1. Left: Porcus material composed of pore $\Omega_{v}$ and skeleton $\Omega_{r}$. Right: Domain $\Omega_{\alpha} \alpha=r, v$ with boundary $\Gamma_{\mathrm{\alpha}}$ and the cutward normal vector $n(x)$.

Another formal proof of (19) is to consider the gradient in real space of $\theta_{\alpha}$

$$
\begin{aligned}
& \nabla \theta_{\alpha}=\nabla\left(\chi_{\alpha} \theta\right)=\theta \nabla \chi_{\alpha}+\chi_{\alpha} \nabla \theta=\chi_{\alpha}(e-E)-\theta(\boldsymbol{n} \delta)_{I_{\alpha}} \\
& =e_{\alpha}-\theta(\boldsymbol{n} \delta)_{\Gamma_{\alpha}}-\chi_{\alpha} E
\end{aligned}
$$

and apply the Fourier transform to the final result. A remark can be made here that $e_{\alpha}(x) \neq \nabla \theta_{\alpha}(x)$ due to the discontinuity of $\theta_{\alpha}$ and of the characteristic function $\chi_{\alpha}$ at $\Gamma_{\alpha}$. Here, $\theta_{\alpha}=\theta$ and $\chi_{\alpha}=1$ inside $\Omega_{\alpha}$ and both vanish $\theta_{\alpha}=0$ and $\chi_{\alpha}=0$ outside $\Omega_{\alpha}$. The spatial derivative of the characteristic function $\chi_{\alpha}$ results in a surface delta term $(\boldsymbol{n} \delta)_{\Gamma_{\alpha}}$ at the boundary $\Gamma_{\alpha}$, as expected.

Now, applying operator $\boldsymbol{R} *$ to the final result in (19) and using (11) yields the result

$$
R * \chi_{\alpha}(E+\nabla \theta)=R * E \chi_{\alpha}-\theta_{\alpha}+\theta_{\alpha}+R *(n \delta)_{\Gamma_{\alpha}} \theta
$$

where $\theta_{\alpha}$ is the average temperature of $\theta_{\alpha}$, or $\theta_{\alpha}=\left\langle\theta_{\alpha}\right\rangle$. Due to the fact that $\theta_{v}+\theta_{r}=\theta$ and $\theta_{v}+\theta_{r}=\theta$, we can eliminate $\theta_{v}$ and obtain the expression for $\theta_{r}$

$$
\begin{aligned}
& \theta_{r}=\theta_{r}+\frac{k_{r}-k_{0}}{k_{0}}\left[\boldsymbol{R} *\left(\boldsymbol{E} \chi_{r}+(\boldsymbol{n} \delta)_{\Gamma_{r}} \theta+\nabla \theta_{r}\right)\right] \\
& -\boldsymbol{R} *\left(\boldsymbol{E} \chi_{\chi_{v}}+(\boldsymbol{n} \delta)_{\Gamma_{v}} \theta\right)
\end{aligned}
$$

Since the two phases $r$ and $v$ share the same boundary, i.e., $\Gamma_{v}=\Gamma_{r}$ but belong to two opposite sides, we have the following properties:

$$
(\boldsymbol{n} \delta)_{\Gamma_{v}}=-(\boldsymbol{n} \delta)_{\Gamma_{r}}
$$

We can note from (25) and especially when $k_{r}=k_{0}$, i.e.

$$
\theta_{r}=\theta_{r}-\boldsymbol{R} *\left(\boldsymbol{E} \chi_{v}+(\boldsymbol{n} \delta)_{\Gamma_{v}} \theta\right)
$$

that the internal temperature field $\theta_{r}$ can be determined from the temperature on the boundary via the term $(\boldsymbol{n} \delta)_{\Gamma}, \theta$. This property renders the approach related to solutions of a Boundary Integral Equation in the periodic setting. 
To derive the equation for $\theta_{r}$, the next step is to express the remaining term $(\boldsymbol{n} \delta)_{\Gamma_{\alpha}} \theta$ as a function of $\theta_{r}$ instead of $\theta$ on the right hand side of Eq. (25). Different from $\theta, \theta_{r}$ is continuous in $\Omega_{r}$ and discontinuous at the boundary $\Gamma_{r}$ where $\theta_{r}=\theta$ inside while $\theta_{r}=0$ outside. Starting from the expression (20) of $\left[(\boldsymbol{n} \delta)_{\Gamma_{\alpha}} \theta\right](\xi)$ in Fourier space, we estimate the surface integral by using a volume integral over a layer of small thickness $\epsilon$ centered at $\Gamma_{\alpha}$ as in Fig. 1, or

$$
\frac{1}{V} \int_{\Gamma_{\alpha}} \theta(x) n(x) e^{-i \xi x} d s=\lim _{\epsilon \rightarrow 0} \frac{1}{V \epsilon} \int_{\Gamma_{\alpha} \times \epsilon} \theta(x) n(x) e^{-i \xi \cdot x} d x
$$

When replacing $\theta$ by $\theta_{r}$, the volume integral in the part outside $\Omega_{\alpha}$ is null while the one within $\Omega_{\alpha}$ is the same as the one using $\theta$. As a result, we obtain the relation

$$
\lim _{\epsilon \rightarrow 0} \frac{1}{V \epsilon} \int_{\Gamma_{\alpha} \times \epsilon} \theta(x) n(x) e^{-i \xi \cdot x} d x=2 \lim _{\epsilon \rightarrow 0} \frac{1}{V \epsilon} \int_{\Gamma_{\alpha} \times \epsilon} \theta_{r}(x) n(x) e^{-i \xi \cdot x} d x
$$

which can be recast into the identity

$$
(\boldsymbol{n} \delta)_{\Gamma_{\alpha}} \theta=2(\boldsymbol{n} \delta)_{\Gamma_{\alpha}} \theta_{r}
$$

This result may be surprising, because $\theta$ and $\theta_{r}$ seem to be characterized by similar values at the boundary. However, the second function is discontinuous at the boundary, explaining why the factor $1 / 2$ appears in (30), similarly to the same factor obtained when writing a boundary integral equation at a boundary point for a solution using the Boundary Element Method [35] or to the factor $1 / 2$ often used for defining the Heaviside function at the discontinuity point. For further convincing of this result, Appendix A produces a mathematically rigorous proof in Fourier space of this equality and a more physical proof in the case of circular inclusions, using known properties of the scalar $\delta$ function. Finally, substituting (30) into (25) yields an equation for $\theta_{r}$

$$
\begin{aligned}
& \theta_{r}=\theta_{r}+\frac{k_{r}-k_{0}}{k_{0}}\left[\boldsymbol{R} *\left(\boldsymbol{E} \chi_{r}+2(\boldsymbol{n} \delta)_{\Gamma_{r}} \theta_{r}+\nabla \theta_{r}\right)\right] \\
& -\boldsymbol{R} *\left(\boldsymbol{E} \chi_{\chi_{v}}+2(\boldsymbol{n} \delta)_{\Gamma_{v}} \theta_{r}\right)
\end{aligned}
$$

In the particular case where the reference material is chosen equal to the matrix $k_{0}=k_{r},(31)$ is reduced to the very simple equation:

$$
\theta_{r}=\theta_{r}-R *\left(E \chi_{v}+2(n \delta)_{\Gamma_{v}} \theta_{r}\right)
$$

The two Eqs. (31) and (32) are equations for $\theta_{r}$ only and can thus be solved by iterations. Again, the constant $\theta_{r}$ can be set arbitrarily, for example $\theta_{r}=0$.

\subsection{Numerical iteration schemes}

We shall present the iteration schemes used to solve the temperature equations (9) and (31). We note that while the scheme based on (9) is essentially the same as the basic scheme based on (5), the scheme for (31) is different due to the decomposition of the domain and the appearance of the boundary term $(\boldsymbol{n} \delta)_{\Gamma} \theta_{r}$.

To solve the original temperature equation (9) with $\theta=0$, one can use the iteration scheme given by the recurrence relation:

$$
\theta^{(n+1)}(x)=R *\left(\delta k / k_{0}\right)\left(\nabla \theta^{(n)}(x)+E\right),
$$

Using the properties of $\boldsymbol{R}$ in (11), we can rearrange the implementation of the recurrence relation by using Algorithm 1 in Fourier space. At convergence, when the difference $\left\|\theta^{(n+1)}(\xi)-\theta^{(n)}(\xi)\right\| \rightarrow 0$, we have $\left\|\boldsymbol{R}(\xi) \boldsymbol{j}^{(\boldsymbol{n})}(\boldsymbol{\xi}) / k_{0}\right\| \rightarrow 0$ and the flux field is also divergence free according to (12). After convergence (step $n_{\text {conv }}$ ), the real field can be recovered by the inverse Fourier transform. The macroscopic flux $J$ can be used to extract the effective property $\boldsymbol{K}^{e}$. The reference material $k_{0}$ is chosen as the average of the minimum and maximum conductivities $k_{\min }, k_{\max }$ found in the system. Such a choice guaranties the optimal convergence of the iteration scheme. 


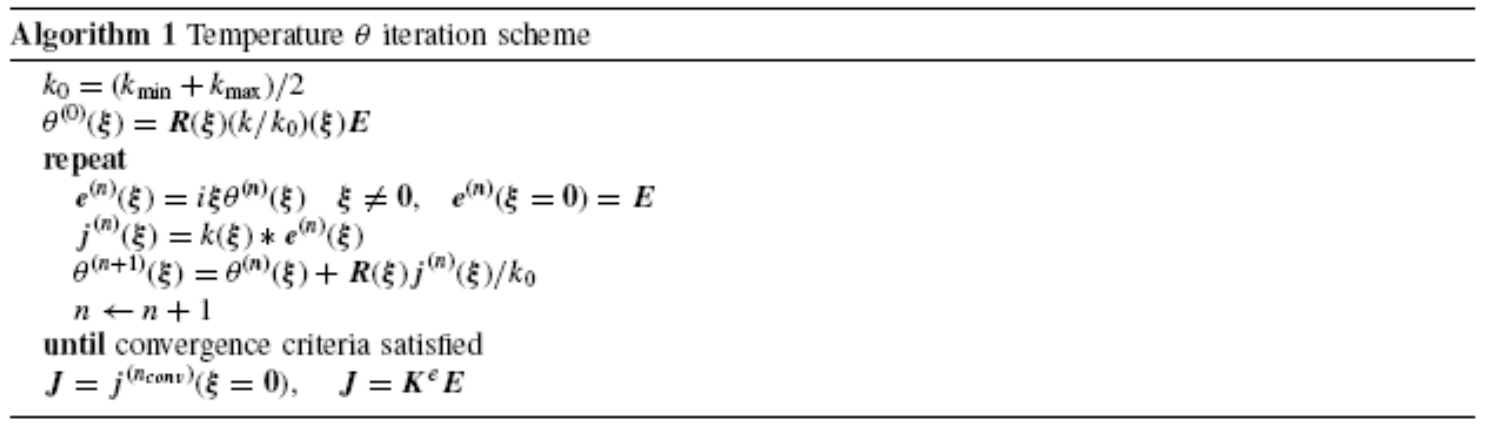

It is possible to show that the above scheme in this form is equivalent to the scheme associated to (5)

$$
e^{(n+1)}(x)=E-P *\left(\delta k / k_{0}\right) e^{(n)}(x)
$$

Algorithm 2 is the well-known original iteration scheme on $E$ described in literature works. In this case, the computation of temperature $\theta$ can be avoided during the iteration process and can be recovered at convergence from $e$.

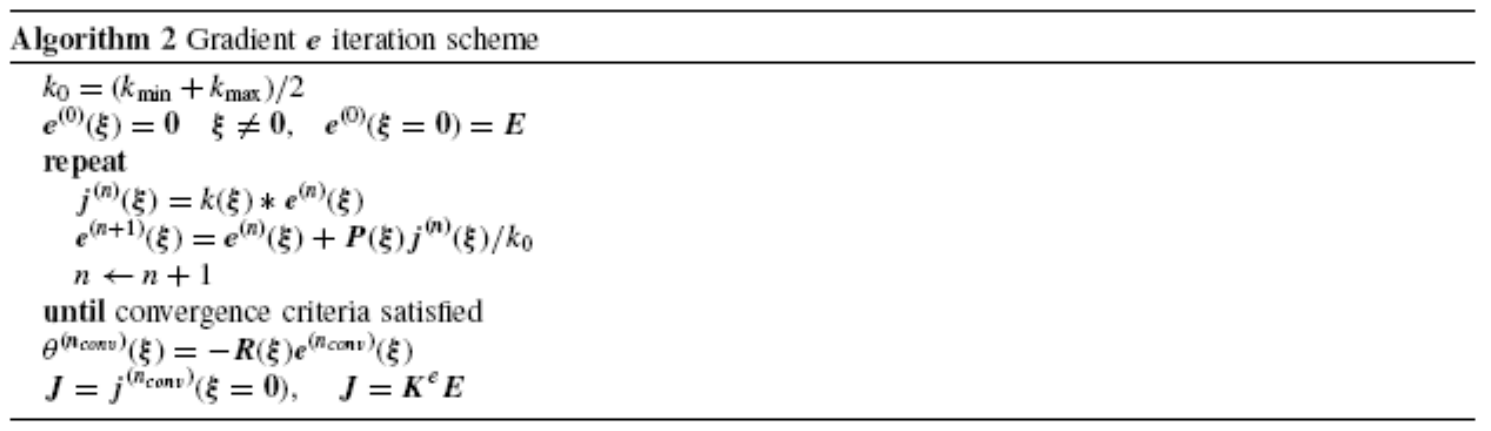

For comparison purpose, we also use the accelerated scheme based on polarization $[6-8,29]$ detailed in Algorithm 3. Based on the polarization variable $\boldsymbol{\tau}$ with prescribed average $T$, the scheme is known for leading to faster convergence rate than the original iteration scheme. However, like other schemes, it also has infinite contrast issues due to the problem of solution uniqueness discussed in the previous section. From Algorithm 3, we can find that at convergence $\left\|\boldsymbol{\tau}^{(n+1)}(\xi)-\tau^{(n)}(\xi)\right\|$, the gradient field is rotation free $\left\|\boldsymbol{Q}(\xi) \boldsymbol{e}^{(n)}(\xi)\right\| \rightarrow 0$ with $\boldsymbol{Q}(\boldsymbol{\xi})=\boldsymbol{I}-\boldsymbol{P}(\boldsymbol{\xi})$ and the flux is divergence free $\left\|\boldsymbol{P}(\xi) j^{(n)}(\xi)\right\| \rightarrow 0$. Those conditions can be used to control the convergence of the scheme.

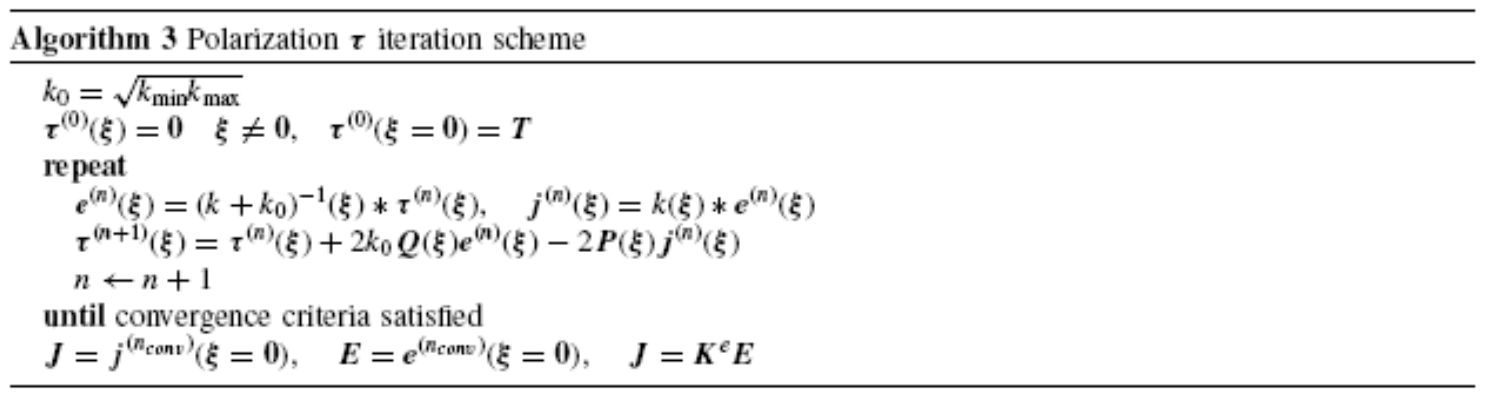

Finally, regarding Eq. (31) for materials containing voids, the associated iterative scheme with $\theta_{r}=0$ can be reformulated in Algorithm 4 using (11). Once again, we can find that at convergence $\left\|\theta_{r}^{(n+1)}(\xi)-\theta_{r}^{(n)}(\xi)\right\| \rightarrow 0$, the flux field is also divergence free due to (12). 


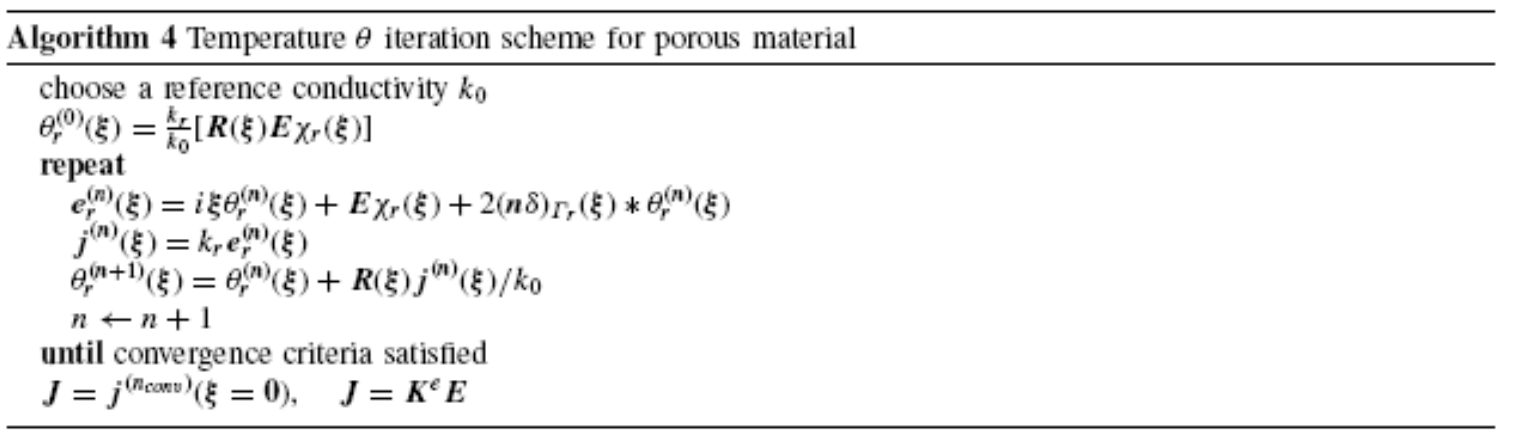

The formulations above are written for continuous variable $\boldsymbol{x}$ and an infinite number of wavevectors $\boldsymbol{\xi}$. Numerically, we discretize $V$ in voxels with resolution of $2 \mathrm{~N} \times 2 \mathrm{~N} \times 2 \mathrm{~N}$ in physical space and limit the wavevectors in the range $-N<n_{i}<N$ with $i=1,2,3$. Since the switching between physical space and Fourier space can be done quickly by the Discrete Fourier Transform algorithm, mathematical operations like the discrete convolution operators $*$ can be affected without difficulties.

To achieve the best accuracy for the last $\theta$ scheme, we use a finer resolution for the Dirac tem $(n \delta)_{\Gamma}(\xi)$ associated with the interface $\Gamma$. Specifically, to evaluate $(\boldsymbol{n} \delta)_{\Gamma}(\xi) * \theta_{r}^{(\boldsymbol{n})}(\boldsymbol{\xi})$, we adopt $-\alpha_{d} N<n_{i}<\alpha_{d} N$ for the special term $(\boldsymbol{n} \delta)_{\Gamma}$ and keeping $-N<n_{i}<N$ for $\theta_{r}^{(n)}(\xi)$ before carrying out the discrete convolution $*$ in Fourier space. While in most applications, the typical value of $\alpha_{d}=2$ yields good results, we also investigate the influence of $\alpha_{d}$ in more detail.

Finally, to control the convergence, three criteria will be considered for comparison: the criterion on the divergence free flux

$$
\left\|i \xi j^{(n)}(\xi)\right\|_{F} /\left(\left|j^{(n)}(0)\right| / L\right) \leq \varepsilon,
$$

the criterion on relative difference of temperature

$$
\left\|\theta_{r}^{(n+1)}(\xi)-\theta_{r}^{(n)}(\xi)\right\|_{F} /\left\|\theta_{r}^{(n+1)}(\xi)\right\|_{F} \leq \varepsilon
$$

and the criterion on relative difference of gradient

$$
\left\|e_{r}^{(n+1)}(\xi)-e_{r}^{(n)}(\xi)\right\|_{F} /\left\|e_{r}^{(n+1)}(\xi)\right\|_{F} \leq \varepsilon
$$

with sufficiently small tolerance $\varepsilon$. For the accelerated scheme based on polarization (see Algorithm 3), we shall use both the divergence of the flux $j$ simultaneously with the curl of the gradient $e$

$$
L\left\|i \xi j^{(n)}(\xi)\right\|_{F} /\left|j^{(n)}(0)\right| \leq \varepsilon, \quad L\left\|i \xi \times e^{(n)}(\xi)\right\|_{F} /\left|e^{(n)}(0)\right| \leq \varepsilon
$$

and the criterion on relative difference

$$
\left\|\tau^{(n+1)}(\xi)-\tau^{(n)}(\xi)\right\|_{F} /\left\|\tau^{(n+1)}(\xi)\right\|_{F} \leq \varepsilon
$$

In this paper, the Frobenius norm $\|_{\cdot \|_{F}}$ for complex tensor is used for the Fourier space, which is equivalent to $L_{2}$ norm in physical space. For example for a scalar function $f$

$$
\|f(\xi)\|_{F}=\sqrt{\sum_{\xi}|f(\xi)|^{2}}
$$

We note that the convergence rate depends on the choice of reference conductivity $k_{0}$. For systems where the conductivity is bounded by $0<k_{\min }<k(x)<k_{\max }<\infty$, the best choice for the original temperature scheme is $k_{0}=\left(k_{\min }+k_{\max }\right) / 2$ and $k_{0}=\sqrt{k_{\min } k_{\max }}$ for polarization scheme. However, for systems containing voids and the new modified scheme, the question is still open and will be examined in the next section.

We also remark that for porous materials, there are special cases where the effective conductivity can vanish, i.e. $j^{(n)}(0) \rightarrow J=0$ but the temperature field $\theta_{r}$ can exist, for example in the case of parallel pores. Since $j^{(n)}(\boldsymbol{0})$ appears in the denominator of (35), this issue would render the convergence criterion impossible to achieve. To 
extend the present temperature scheme to those cases, we need to replace $j^{(n)}(0)$ by $k_{r}|E|$ where $E$ is the driving force specified from the beginning of the schemes. The convergence criteria (35) now become

$$
\left\|i \xi j^{(n)}(\xi)\right\|_{F} /\left(k_{r}|E|\right) \leq \varepsilon,
$$

\section{Applications to homogenization problems}

\subsection{General description of systems, phase geometry and form factors}

To test the algorithm established previously, we shall study 2D systems in the plane $1-2$ constituted of a square unit cell with dimensions $V=L_{1} \times L_{2}$ and $L_{1}=L_{2}=L=1$. We will study cases where the geometries $\chi_{\alpha}(x)$ can be smooth (ellipse) and non-smooth (polygons) and combinations (see Fig. 2). In those cases, the form factor $\chi_{\alpha}(\xi)$ is explicit. For example, if the phase $\alpha$ is an ellipse of semi axes $R_{1}, R_{2}$ centered at $c$, we have [31,32]

$$
\chi_{\alpha}(\xi)=\frac{2 S}{V} \frac{J_{1}(\eta)}{\eta} e^{-i \xi c}, \quad \eta=\sqrt{\xi_{1}^{2} R_{1}^{2}+\xi_{2}^{2} R_{2}^{2}}, \quad S=\pi R_{1} R_{2}
$$

with $J_{1}$ is the first order Bessel function of first kind. For polygons with corner $c_{1}, c_{2}, c_{3}, \ldots, c_{J}$ in counter clock wise direction $[29,30]$

$$
\begin{aligned}
& \chi_{\alpha}(\xi)=\frac{\hat{i}_{3} \times \xi}{i V \xi^{2}} \cdot \sum_{j=1}^{J}\left(c_{j}-c_{j-1}\right) \operatorname{sinc}\left[\xi \frac{\left(c_{j}-c_{j-1}\right)}{2}\right] e^{-i \xi \frac{c_{j+c_{j-1}}}{2}} \\
& S=\frac{1}{2} \hat{i}_{3} \cdot \sum_{j=1}^{J} c_{j-1} \times c_{j}, \quad c_{0} \equiv c_{J}
\end{aligned}
$$

where $\hat{i}_{3}$ is the unit vector normal to the working plane $\left(\hat{i}_{1}, \hat{i}_{2}\right)$.

Another case of interest is when the void phase is obtained from pixel images. The latter can be considered as a group of identical squares of dimensions $L /(2 N) \times L /(2 N)$ with centers located at the grid points $c_{1}, c_{2}, \ldots, c_{J}$. The exact expression of the form factor is given by

$$
\chi_{\alpha}(\xi)=\left[\frac{L}{2 N}\right]^{2} \sum_{j=1}^{J} \operatorname{sinc}\left[\frac{\xi_{1} L}{2 N}\right] \operatorname{sinc}\left[\frac{\xi_{2} L}{2 N}\right] e^{-i \xi \cdot c_{j}}
$$

We note that this formula is different from the Discrete Fourier Transform of $\chi$

$$
\chi_{\alpha}^{D F T}(\xi)=\left[\frac{L}{2 N}\right]^{2} \sum_{j=1}^{J} e^{-i \xi c_{j}}
$$

by the tem sinc. On the other hand, the void phase can also be treated as a set of polygons with vertices being the centers of the boundary cells and whose form factor is given by (42). All those three possibilities will be investigated.

The problems are solved with the resolutions $N=128,256,512$ and results will be compared with the solutions using COMSOL, a standard Finite Element Method software and with closed form solutions. We shall examine both the local field and the effective property obtained by the method. The latter is obtained from the relation between $J$ and $E$.

\subsection{Convergence issues of basic and accelerated schemes at high contrast ratio}

As mentioned earlier, the temperature scheme is directly derived from the gradient scheme and is expected to have the same eigenvalues, spectral radius and convergence behaviors. Like the basic and polarization schemes, the scheme is not guaranteed to converge for the infinite contrast case, i.e. $k_{\max } / k_{\min }=\infty$. In this subsection, we shall study briefly the accuracy and the convergence behavior of these schemes.

We consider the case of a circular inclusion of radius $R=0.3$ located at the center of the unit square $V$ and embedded within the matrix. The matrix conductivity is fixed as $k_{1}=1$ and the inclusion conductivity $k_{2}$ is varied. The reference conductivity is $k_{0}=\left(k_{1}+k_{2}\right) / 2$ for the basic schemes and $k_{0}=\sqrt{k_{1} k_{2}}$ for the accelerated polarization 

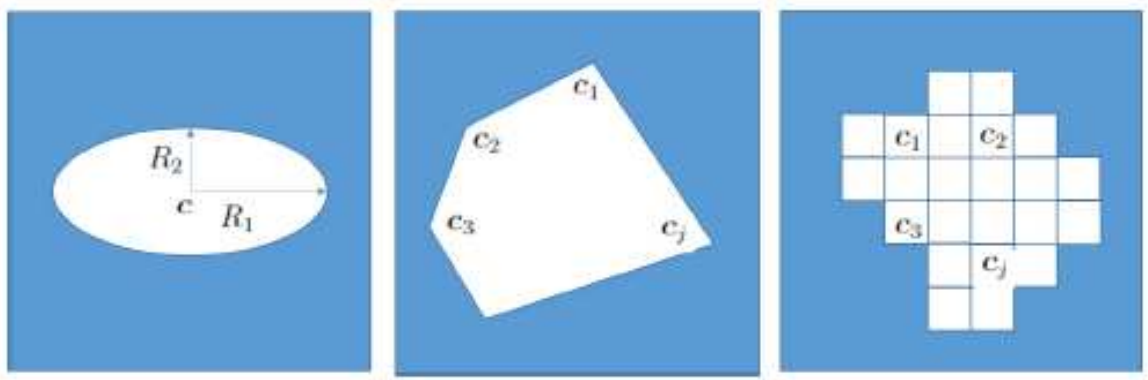

Fig. 2. Geometries of pore considered in the paper. Left: ellipse with semi axes $R_{1}, R_{2}$ sentered at $c$. Center polygons with comers $c_{1}, c_{2}, c_{3}, \ldots, c j$ in counter clock wise direction. Right: Group of squares with oenters $c_{1}, c_{2}, c_{3}, \ldots, c j$ obtained from pixel images.

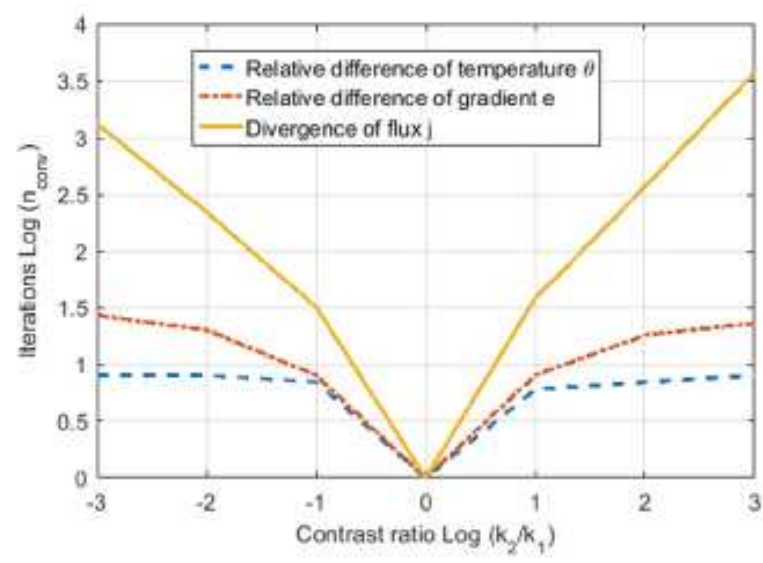

Fig. 3. Comparisons of corwergence criteria used in basic schemes.

scheme. The unit macroscopic loading value is applied along the horizontal direction $E=\hat{i}_{1}$ (basic schemes) and $T=\hat{i}_{1}$ (polarization schemes). To achieve the best convergence behavior, we adopt the maximal resolution $N=512$ and the tolerance $\varepsilon=0.001$.

From Figs. 3, 4, we can find that the scheme is sensitive to the choice of criteria. Among the relative difference criteria, the use of temperature results leads the program to terminate much more earlier than for the gradient. On the other hand, the relative difference criterion does not guarantee the divergence free criterion, which is important in the resolution of conduction problem and seems to be a very demanding criterion. At high contrast ratio, say $k_{2} / k_{1}>10$, the iteration curve of divergence free criteria increases linearly in the Log-Log plot. This behavior agrees with previous works on this aspect (see e.g. [12]). Especially, in the case $k_{2}=0$ (void), numerical tests show that the schemes do not converge. The use of the accelerated scheme only improves the convergence for a finite contrast (see Fig, 4) but cannot solve the case of infinite contrast. As seen later, this bad convergence behavior for porous material is improwed by the temperature scheme developed in this work.

\subsection{Performance of temperature scheme for porous material}

To test the new temperature scheme devoted to a porous material, we take as a first example the same system as in the previous subsection but use the value $k_{2}=0$ for inclusion and $k_{r}=k_{1}=1$ for the matrix and use Algorithm 4 to solve the problem. The resolution $N=128$ and the tolerance $\varepsilon=0.001$ are adopted in this case.

In this section, with a chosen reference material $k_{0}=k_{r}=1$, the scheme converges very quickly. It takes 15 iterations to satisfy all the criteria, including the demanding condition on the flux divergence. From the first 


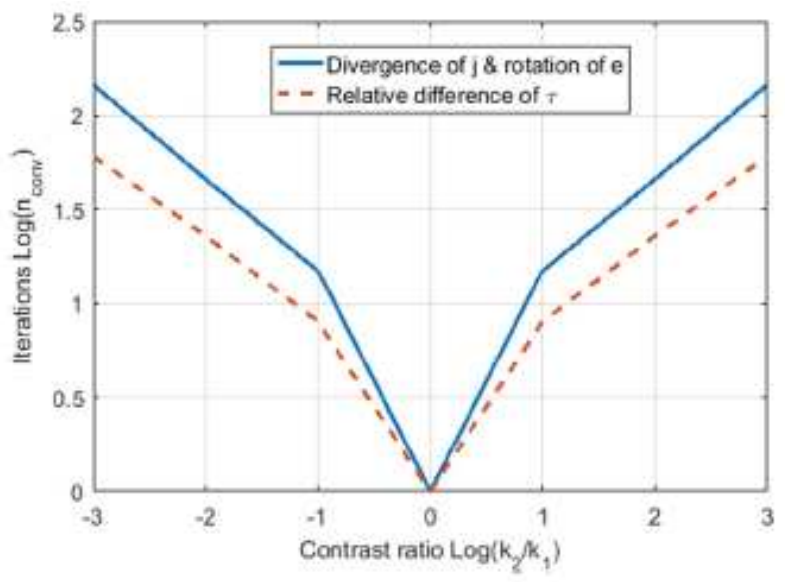

Fig. 4. Comparisons of convergence criteria used in the acoelerated scheme.
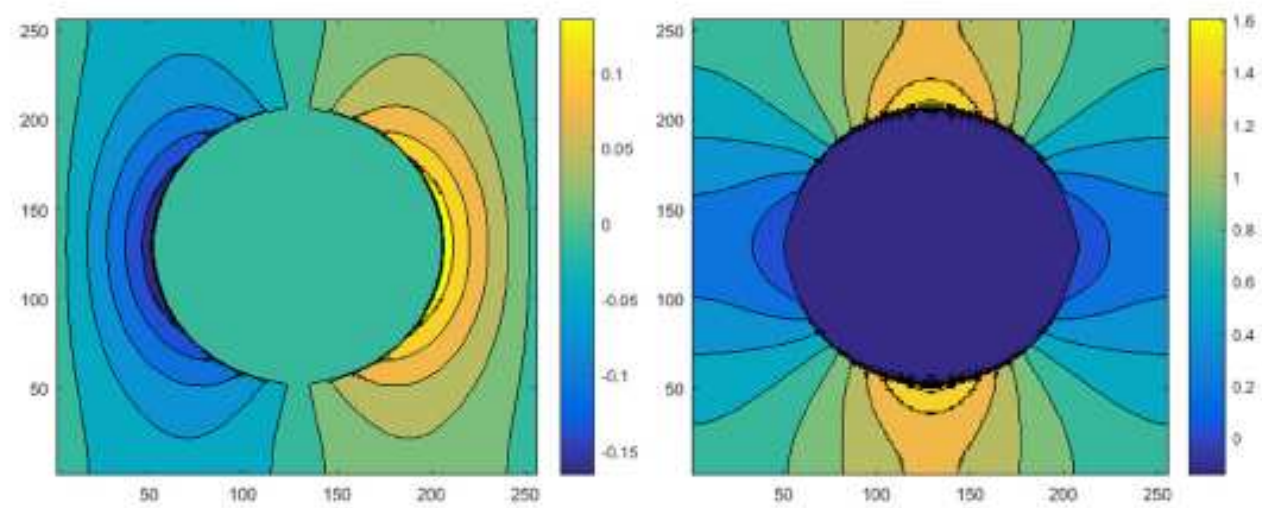

Fig 5. Filled contourplot of periodic temperature field $\theta_{r}(x)$ and the gradient field $e_{r}(x)$ along direction 1 in the regular conductive zone.

qualitative inspection in Fig. 5, we can find that $\theta_{r}$ vanishes in the pore. It is also symmetrical with respect to axis 1 and antisymmetrical with respect to axis 2 . The gradient components along 1 of $\boldsymbol{e}_{r}$ which vanish in the pore, also possess the necessary symmetry properties with respect to axes 1 and 2 .

Next, we look at the local fields and compare the results with those of FEM. From the contour plot in Fig. 6 , we find that the modified scheme compares well with FEM simulations. Some fluctuations are found near the void boundary, which are common features of methods based on Fourier series (i.e. the Gibbs phenomenon). However, the effective conductivity $k_{e}=0.5589$ is in excellent agreement with the value $k_{e}=0.5585$ by FEM.

We also do tests with a polygonal void. In this case, a diamond void with diagonals of length 0.8 and 0.4 is located at the center of $V$. We find that the non-smooth inclusion form has a significant effect on the convergence rate. It requires 18 iterations to satisfy the relative difference criteria and 40 iterations for divergence free flux criteria. The obtained results are also satisfactory both in terms of local field (see Fig. 17) and effective property, $k_{e 1}=0.7838$ (FFT) vs $k_{e 1}=0.7835$ (FEM) along direction 1 (see Fig. 7).

\subsection{Accuracy assessment of solution fields}

In Section 3.3, we have examined qualitatively the solution fields and the accuracy of the resulted effective properties produced by the temperature scheme. The present section will be devoted to a more detailed comparison 

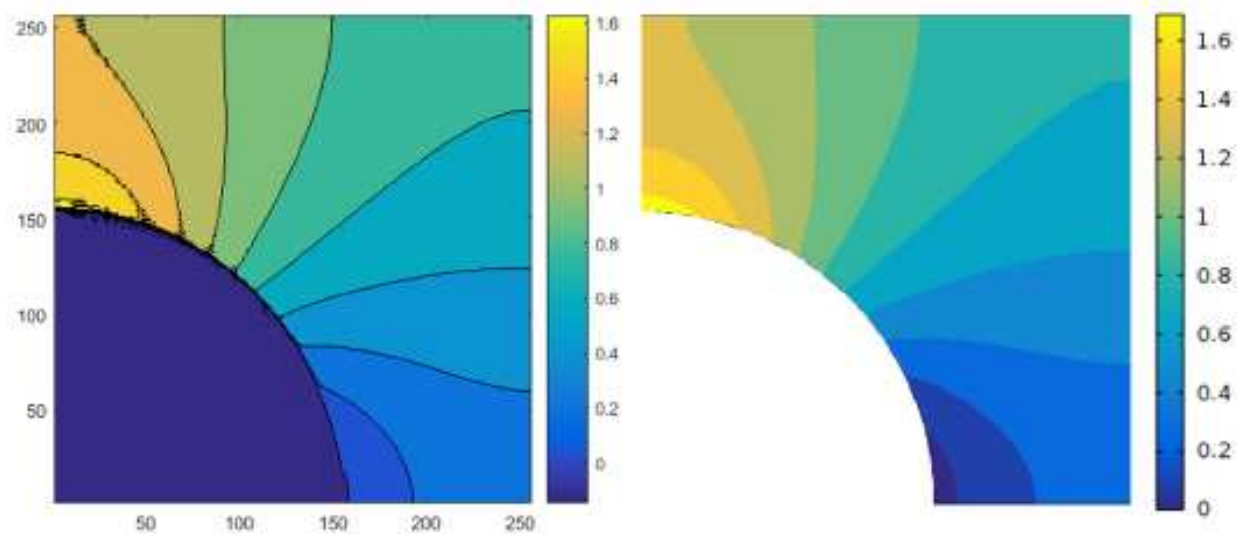

Fig. 6. Filled contour plot with 10 levels of gradient field $e_{r}(x)$ along direction 1 by FFT method (left) and FEM (right) for circle void. Due to the symmetry, only a quarter of the model is shown.
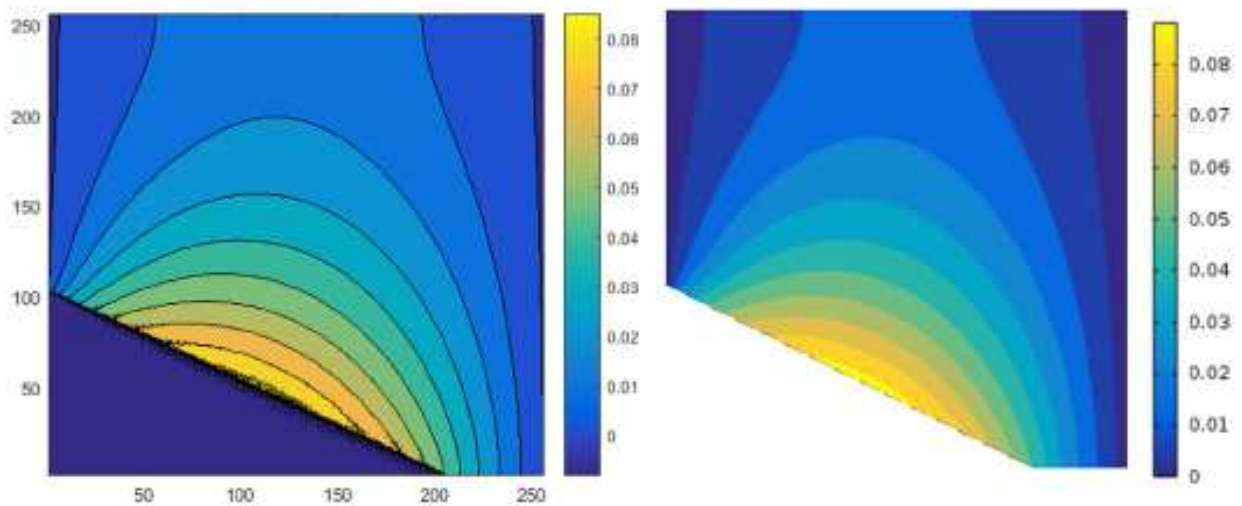

Fig. 7. Contourplot with 10 levels of temperature field $\theta_{r}(x)$ by FFT method (left) and FEM (right) for diamond void. Due to the symmetry, only a quarter of the model is shown.

of the whole field which is crucial for judging the quality of the method. We choose the cases of cylinder and square pores whose benchmark solutions in literature are available.

First, let us look into the same case of cylinder pore of radius 0.3 as before. The reference solution for temperature field $\theta_{r}$, based on the classical Rayleigh's method [36] described in Appendix C for the case of voided inclusions, is used for comparison purpose. As seen in Fig. 8, we find that the FFT solutions are in excellent agreement with the Rayleigh solution. The temperature values $\theta_{r}$ vanish inside the pore and match the values computed by the Rayleigh method for the solid phase. Near the interface pore-solid, some undershoots and overshoots are observed, which can be explained by the Gibbs phenomenon. But globally, despite the localized fluctuation, the relative errors between the two solution fields are small and decrease when increasing the resolution parameter $N$. We note that although the solution $\theta_{r}$ computed by the present FFT method is very small in the void but still different from the theoretical value 0 . Different from the skeleton field error $\left\|\theta_{r}^{F F T}-\theta_{r}^{\text {Rayleigh }}\right\|^{\Omega_{r}} /\left\|\theta_{r}^{\text {Rayleigh }}\right\|^{\Omega}$, the whole field error $\left\|\theta_{r}^{F F T}-\theta_{r}^{\text {Rayleigh }}\right\|^{V} /\left\|\theta_{r}^{\text {Rayleigh }}\right\|^{V}$ also accounts for the non vanishing void field produced by the numerical method. The norm in real space for a continuous function $f$

$$
\|f(x)\|^{V}=\frac{1}{2 N} \sqrt{\sum_{x^{i} \in V}\left|f\left(x^{i}\right)\right|^{2}}, \quad\|f(x)\|^{\Omega_{r}}=\frac{1}{2 N} \sqrt{\sum_{x^{i} \in \Omega_{r}}\left|f\left(x^{i}\right)\right|^{2}}
$$

is used in this case with $x^{i}$ being the $(2 N)^{2}$ grid points. 

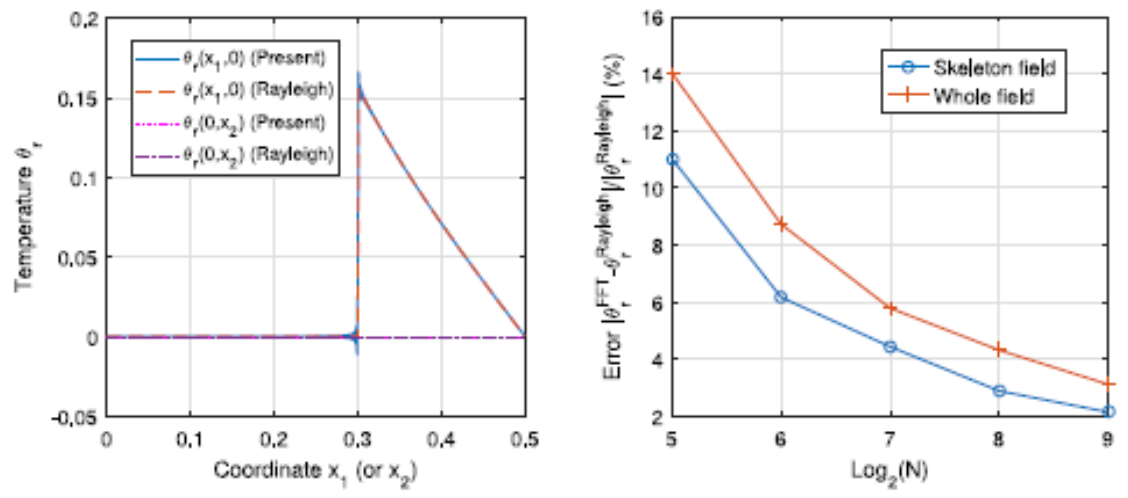

Fig. 8. Left: Temperature profiles along $x_{1}$ and $x_{2}$ axes obtained by the present FFT method with $N=512$ and Rayleigh method for circular pore. Right: Relative error (in percentage) between the two solution fields $\left\|\theta_{r}^{F F T}-\theta_{r}^{\text {Ragieigh }}\right\| /\left\|\theta_{r}^{R a j i e i g h}\right\|$.

Using these criteria in the right part of Fig. 8 shows that the accuracy of the skeleton field is better than the accuracy of the whole field. Next, we consider a problem with square holes whose analytical solution has been obtained by Obnosov [37] and described in Appendix B. In this example, the square hole has dimensions $0.5 \times 0.5$ and has the same center as the unit cell. The reference solutions are given for the gradient fields $\boldsymbol{e}_{\boldsymbol{r}}$. We examine the gradient profiles along the horizontal slice at $x_{2}=0$ and vertical slice at $x_{1}=1 / 4$. From Fig. 9, we find that the FFT solution is in excellent agreement with the Obsonow solution. Again, some localized fluctuation near the interface can be seen, but it does not affect the global accuracy of the FFT solution. We note that due to the singularity of $\boldsymbol{e}_{r}$ at the square corner and the discontinuity of $e_{r}$ on the square edges, we exclude the region of thickness $e=0.01$ centered at the square edges before evaluating the whole field error between the FFT and Obsonov solution. As seen in Fig. 10, the global error is small, confirming the performance of the method for this case. Like for the cylinder case, the error within the skeleton phase is smaller. In addition, at high resolution, the difference between the error within the skeleton field and the one for the whole field is small.

\subsection{Influence of reference conductivity and microstructure on the convergence}

In the previous section, we have chosen the reference material conductivity equal to that of the matrix $k_{0}=k_{r}$. The choice yielded good convergence behavior and results in terms of local field and effective property. In this section we shall address the question about the convergence condition. In addition to the two previous examples on circular and diamond pores, to study the interaction of pores, we consider two more examples where the distribution of circular pores and triangle, quadrilateral pores is relatively dense, i.e. the pores are close to each other. The first sample is constituted of 4 triangles and 2 quadrilaterals with porosity 0.51 and the second sample is made of 6 circular pores of different diameters with porosity 0.46 (see the inset figures of Fig. 12). The demanding criteria on the flux divergence are used to control the convergence.

From Figs. 11 and 12, we find a common convergence behavior. The scheme converges very slowly when $k_{0}<k_{r}$ and even diverges at low $k_{0} / k_{r}$ value. The optimal $k_{0} / k_{r}$ ratio is around 1.2 but not at $k_{0}=k_{r}$. At higher ratios, the required number of iterations increases again, but the computation still performs better than for small ratios. When many pores are present, their interaction requires more iterations to achieve the convergence and the system of circular pores converges better than the system of polygonal pores. For example, for the pack of mixture of 6 circular pores, it requires a minimum of 27 iterations while for the mixture of 6 triangles and quadrilaterals, the minimum value is 240 . This effect suggests that the microstructure and the choice of the reference conductivity are crucial for the optimal convergence of the problem.

\subsection{Pixel images and resolution study}

In Section 3.1, the characteristic functions of the void phases are provided via their Fourier Transforms, i.e. the analytical form factors. The polygon shape is also general and can be used to approximate most geometrical forms. 

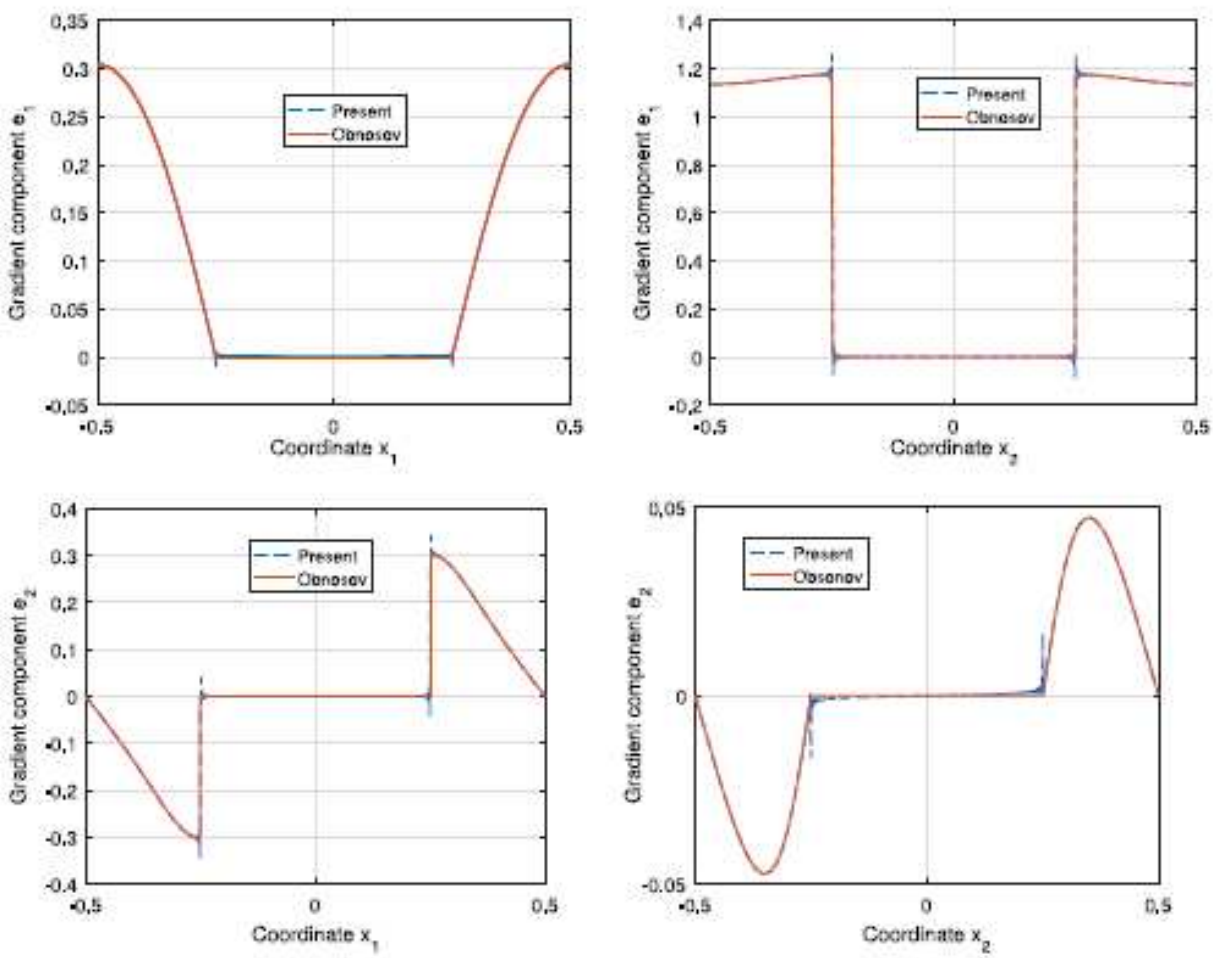

Fig. 9. Comparison between FFT and analytical solution by Obsonov for square voids of sides 0.5 . The variation of gradient components $e_{1}$ and $e_{2}$ is plotted in two upper and two lower figures.

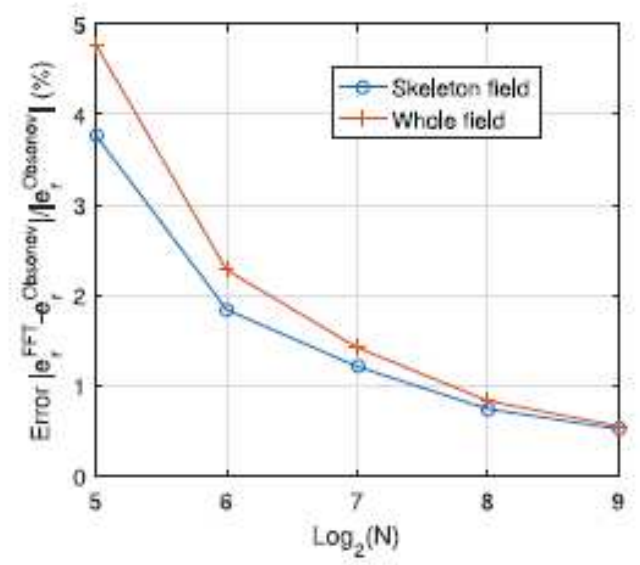

Fig 10. Relative error (in peroentage) between the two solution fields $\left\|e_{r}^{F F T}-e_{r}^{\text {Obonov }}\right\| /\left\|e_{r}^{\text {Obsonov }}\right\|$.

For example, in the case of pixel images frequently used in tomography applications, an approximate polygon can be constructed by connecting the centers of the boundary voxels. The boundary voxels can be detected by standard image processing techniques and Fig. 13 shows an example of a circular pore of radius $R=0.3$. The major advantage of using such a polygonal approximation over the square boundary of the voxels is that the normal vectors $\boldsymbol{n}$ on the boundary associated to the surface Dirac term $(n \delta)_{\Gamma}$ vary less abruptly and minimize the singularity effect at the corners. 


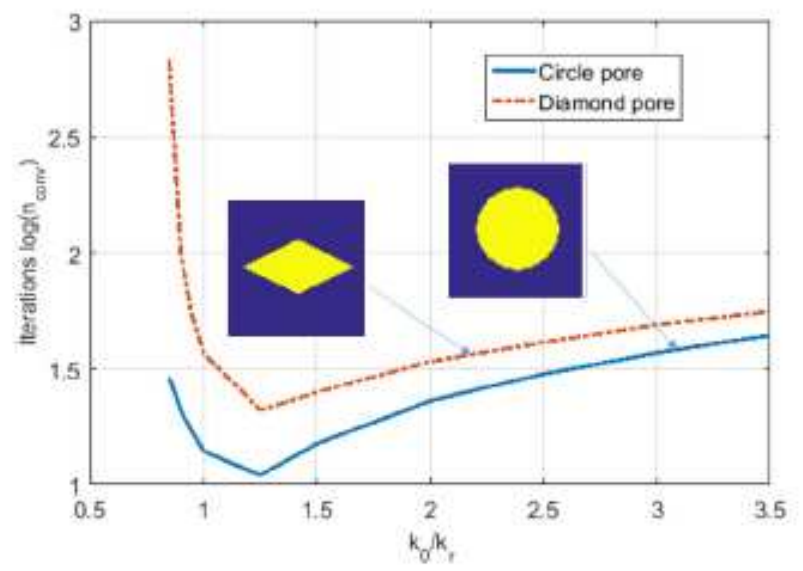

Fig. 11. Influence of reference conductivity $k_{0}$ on the convergence. Case of single circke and diamond pore.

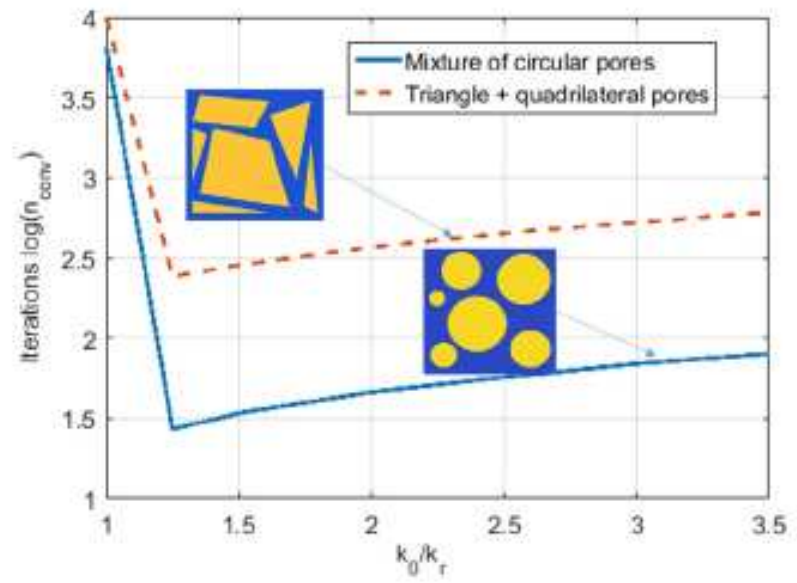

Fig. 12. Influenee of reference conductivity $k_{0}$ on the corvergence. Case of multiple pores (mixture of circular pores vs mixture of polygonal pores).
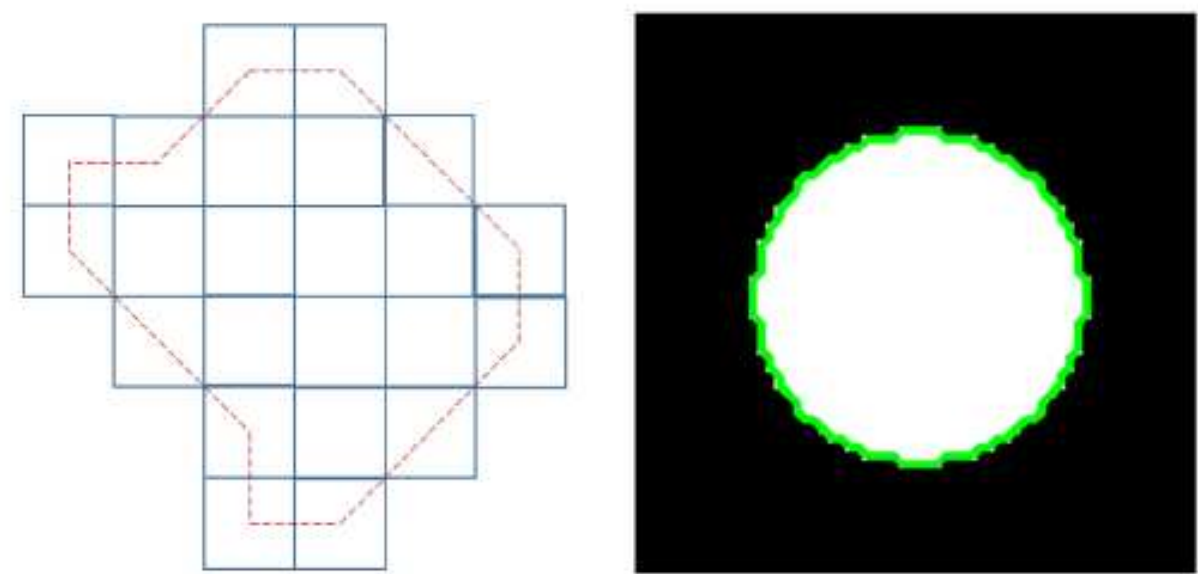

Fig 13. Left: Detection of boundary of a pore from a pixel image and approximation with polygon. Right: Application to circular pore. 


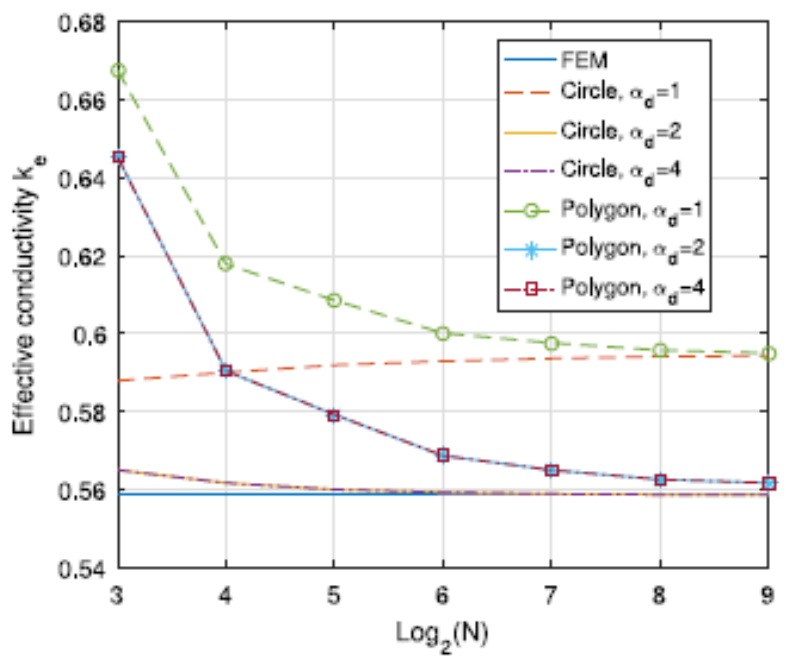

Fig. 14. Effective properties computed by FFT based methods for a circle with radius $R=0.3$. The pixel images approximated by polygons is compared with the exact circk.

Let us recall that the present method is based on the surface Dirac distribution $(\boldsymbol{n} \delta)_{\Gamma}$ which requires a finer resolution in Fourier space, $\alpha_{d} N$ versus the base resolution $N$ for the skeleton phase $\chi_{r}$ and other unknowns $j, e_{r}$. To study how those factors influence the scheme, different resolution parameters $N=8,16,32,64,128,256$ and 512 are used. The refining resolution coefficient $\alpha_{d}$ corresponding to the delta term is also varied from 1 to 4 .

As seen in Fig. 14, using analytical form factors for the circle and its approximate polygon yields already convergent results at $\alpha_{d}=2$. While adopting the same base resolution $\alpha_{d}=1$ is clearly not accurate enough, the curves corresponding to $\alpha_{d}=2$ and $\alpha_{d}=4$ coincide perfectly and decrease smoothly to the FEM results. The results associated to the polygonal approximation show the same behavior but converge more slowly and are slightly less accurate than the results based on the circle form factor. However the difference between the results related to two circle and polygon curves is very small, less than $1 \%$ for the resolution $N=128$.

We find that using the polygon approximation and the associated form factor performs much better than the pixel form factors (exact or DFT). By fixing $N=128$ and increasing the refining coefficient $\alpha_{d}$, the exact and DFT pixel form factors converge very slowly (see Fig. 15). Even for very high value $\alpha_{d}=16$, the difference from the exact result is still considerable. The circle and the polygon smoothing curves converge at $\alpha_{d}=2$ and are both close to the exact results. It suggests that the polygons capture better the variation of the void boundary than the square grid.

As a final remark, the refining resolution factor $\alpha_{d}$ associated to $(n \delta)_{\Gamma}$ term does require an additional memory storage and computation time when compared with other methods dealing with nonporous material. However, when coupling with form factors, it yields superior results in both local fields and effective properties. It is known that methods based on pixel description can predict the effective properties but generate artificially corners and associated singularities and other undesirable effects. Those artificial corners have the same characteristic size as the basic grid spacing. If we want to treat properly the local fields, it is also necessary to adopt a finer grid in addition to the basic grid.

\subsection{Connected pores across the unit cell}

Up to now, only compact pores were studied. However, the solution method being based on an interface operator, it is important to check that the method can also be used with pores crossing the porous cell, when the interface crosses the boundary of the periodic cell. In a first example, we study a pore of height $h$ along direction $x$ centered 


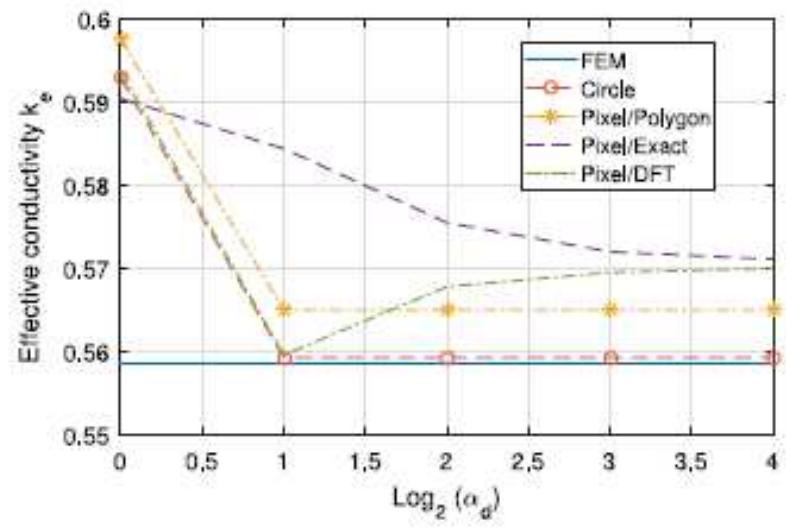

Fig. 15. Effective properties for circular pore obcained with circle form factor and pixel based (polygon, exact and DFT) form factor. The base resolution is fixed at $N=128$.
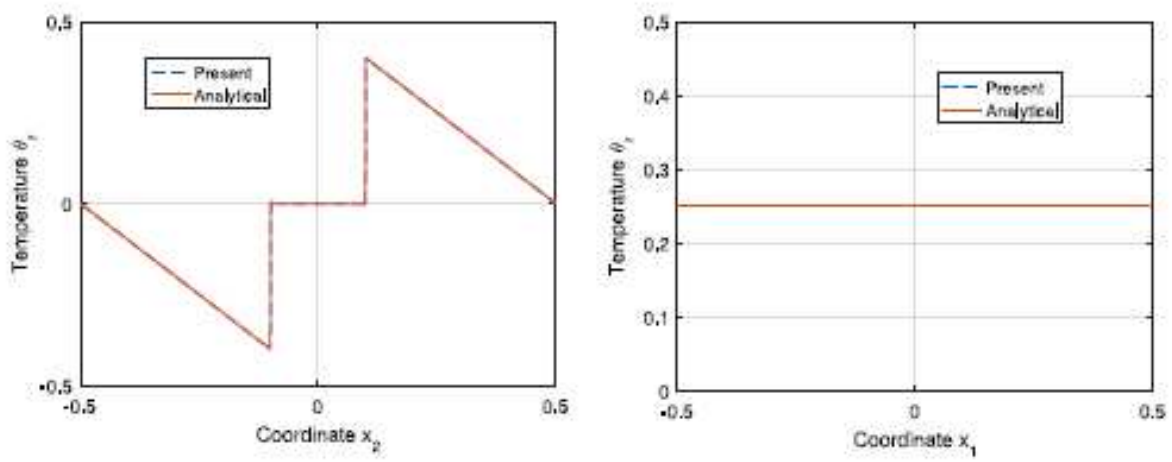

Fig. 16. Temperature profile $\theta_{r}\left(0, x_{2}\right)$ (left) and $\theta_{r}\left(x_{1}, 0.25\right)$ (right) for the straight pore with $E_{1}=0, E_{2}=1$ in comparison with analytical solution.

at our unit cell. The analytical solution for the above system is given below

$$
\begin{array}{ll}
\text { For } E_{1} \neq 0, E_{2}=0: & \theta_{r}(x)=0 \forall x \\
\text { For } E_{1}=0, E_{2} \neq 0: & \theta_{r}(x)=0 \text { for }\left|x_{2}\right|<h / 2 \\
& \theta_{r}(x)=E_{2}\left(1 / 2 \operatorname{sign}\left(x_{2}\right)-x_{2}\right) \text { for } h / 2<\left|x_{2}\right|<1
\end{array}
$$

Those results yield an effective conductivity corresponding with Voigt and Reuss bounds $k_{e 1} / k_{0}=(1-h)$ and $k_{e 2} / k_{0}=0$.

Tests on the case $h=0.2$ show that, at convergence, the FFT results are in excellent agreement with analytical results. When $E_{1} \neq 0, E_{2}=1$, the temperature field is small. When $E_{1}=1, E_{2} \neq 0$, the temperature is linear in each range $[h / 2,1 / 2]$ and $[-h / 2,-1 / 2]$ with the maximal and minimal values being 0.4 and -0.4 (see Fig. 16). The code converges after 1 and 37 iterations for each case. The effective conductivity is exactly identical to the analytical values $k_{e 1} / k_{0}=0.8$ and $k_{e 2} / k_{0}=0$.

In the second example, we study a zigzag pore of height $h=0.2$ (see Fig. 17). The center line connects six points of coordinates $(-0.5,-0.25),(-0.25,0.25),(0.25,0.25),(0.25,-0.25)$ and $(0.5,-0.25)$. The effective conductivity $k_{e 1}=0.333$ ( 26 iterations) can be compared with 0.328 by FEM and $k_{e 2}=0$ ( 134 iterations). Thus, the difference in $k_{e 1}$ is less than $2 \%$ for the resolution $N=128$ and is further improved for higher resolution.

The above two examples show the applicability of the method to a pore network crossing the periodic cell, which is frequently encountered in practice. 

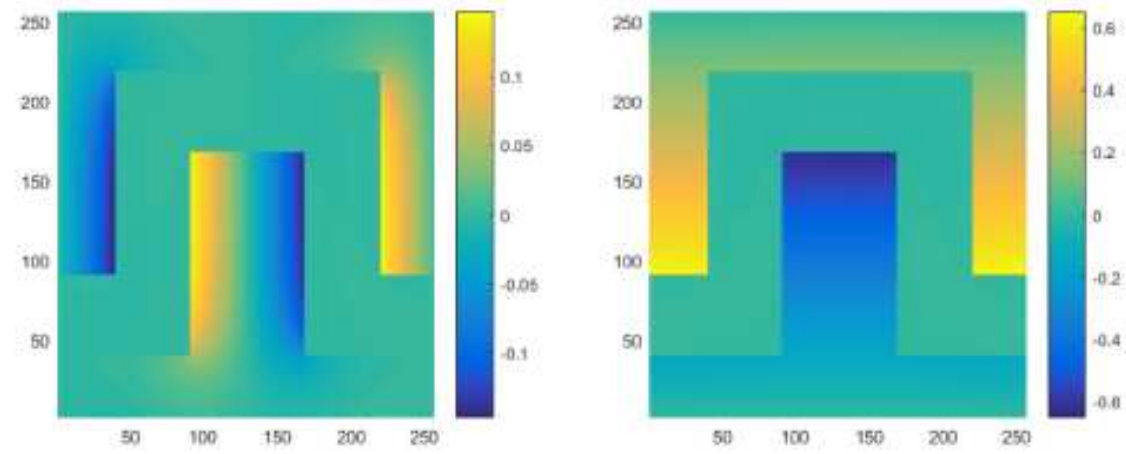

Fig. 17. Temperature field $\theta_{r}(x)$ for the zigzag pore with $E_{1}=1, E_{2}=0$ (kft) and $E_{1}=0, E_{2}=1$ (right).

\section{Conclusion}

In this paper, we have developed a FFT based numerical scheme to compute the effective conductivity of porous materials. To avoid the convergence issues due to the nonuniqueness of the full field solution, we reformulate the problem using the temperature field in the skeleton as an unknown variable. In the newly derived governing equation, the internal temperature field can be computed from the value on the pore boundary. This feature gives the present scheme some similarity with the methods based on Boundary Integral Equations.

When employing iterative resolution schemes, examples show that the new scheme converges very quickly and yields both accurate effective conductivity and satisfying local fields. We also have carried out a convergence study for different types of pore microstructures including circle and polygonal pores and their mixtures. Useful information on the convergence behavior is obtained to help determining the optimal reference conductivity value ensuring the best convergence of the scheme.

\section{Declaration of competing interest}

The authors declare that they have no known competing financial interests or personal relationships that could have appeared to influence the work reported in this paper.

Appendix A. Proof of identity (30)

\section{A.1. Proof in Fourier space}

We shall demonstrate identity (30) by showing that

$$
2(\boldsymbol{n} \delta)_{\Gamma_{\mathrm{e}}}(\boldsymbol{x}) \chi_{r}(\boldsymbol{x})=(\boldsymbol{n} \delta)_{\Gamma_{\alpha}}(\boldsymbol{x})
$$

From (22), it is equivalent to prove the identity in Fourier space

$$
2\left[i \xi \chi_{r}(\xi)\right] * \chi_{r}(\xi)=i \xi \chi_{r}(\xi)
$$

Using the definition of the discrete convolution (4) and after some calculation, we have

$$
\begin{aligned}
& {\left[i \xi \chi_{r}(\xi)\right] * \chi_{r}(\xi)=i \sum_{\xi^{\prime}}\left(\xi-\xi^{\prime}\right) \chi_{r}\left(\xi-\xi^{\prime}\right) \chi_{r}\left(\xi^{\prime}\right)} \\
& =i \xi \sum_{\xi^{\prime}} \chi_{r}\left(\xi-\xi^{\prime}\right) \chi_{r}\left(\xi^{\prime}\right)-\sum_{\xi^{\prime}} \chi_{r}\left(\xi-\xi^{\prime}\right) i \xi^{\prime} \chi_{r}\left(\xi^{\prime}\right) \\
& =i \xi\left(\chi_{r} \chi_{r}\right)(\xi)-\chi_{r}(\xi) *\left[i \xi \chi_{r}(\xi)\right] \\
& =i \xi \chi_{r}(\xi)-\left[i \xi \chi_{r}(\xi)\right] * \chi_{r}(\xi)
\end{aligned}
$$

which leads to (A.2). To arrive at the last line of (A.3), we use the property of the characteristic function in real space $\chi_{r}(x) \chi_{r}(x)=\chi_{r}(x)$ and the fact that the convolution operator $*$ commutes. 


\section{A.2. Proof in real space for circular inclusion}

As a complement to the previous demonstration, we examine the case of circular inclusions of radius $R$, and show that in this case, (29) is a consequence of a classical property of scalar delta function. The polar coordinate system $(\rho, \alpha)$ will be adopted. For any function $\varphi(\rho, \alpha)$, we have

$$
\begin{aligned}
& \left\langle(\boldsymbol{n} \delta)_{\Gamma} \theta(\rho, \alpha), \varphi(\rho, \alpha)\right\rangle=R \int_{\Gamma} \theta(R, \alpha) \varphi(R, \alpha) \boldsymbol{n}(\alpha) d \alpha \\
& =R \int_{\Gamma}\left\langle\delta^{\prime}(\rho-R), \theta(\rho, \alpha) \varphi(\rho, \alpha) n(\alpha)\right\rangle d \alpha=\left\langle\delta^{\prime}(\rho-R), \mathbf{T}(\rho)\right\rangle
\end{aligned}
$$

with $\mathbf{T}(\rho)$ being the integral

$$
\mathbf{T}(\rho)=R \int_{\Gamma} \theta(\rho, \alpha) n(\alpha) \varphi(\rho, \alpha) d \alpha .
$$

and $\delta^{\prime}$ being the scalar delta function. The latter can be classically defined as

$$
\delta^{\prime}(\rho-R)=\lim _{a \rightarrow 0} \frac{1}{2 \sqrt{\pi a}} e^{-(\rho-R)^{2} / 4 a}
$$

Applying the above definition of scalar delta function and making use of the continuity of $\mathbf{T}, \theta$ and $\varphi$ at vicinity of $\rho=R$, we obtain

$$
\begin{aligned}
& \left\langle(\boldsymbol{n} \delta)_{N} \theta, \varphi\right\rangle=\lim _{a \rightarrow 0} \int_{0}^{\infty} \frac{1}{2 \sqrt{\pi a}} e^{-(\rho-R)^{2} / 4 a} \mathbf{T}(\rho) d \rho \\
& =\mathbf{T}(R) \cdot \lim _{a \rightarrow 0} \int_{0}^{\infty} \frac{1}{2 \sqrt{\pi a}} e^{-(\rho-R)^{2} / 4 a} d \rho=\mathbf{T}(R)
\end{aligned}
$$

We consider now the discontinuous function $\theta_{r}=\chi_{r} \theta$ with $\chi_{r}$ being the characteristic function of the circular inclusion. The latter is equivalent to a scalar Heaviside function $\chi_{r}(\rho, \alpha)=H(R-\rho)$, leading to

$$
\begin{aligned}
& \left\langle(\mathbf{n} \delta)_{\Gamma} \theta_{r}, \varphi\right\rangle=\lim _{a \rightarrow 0} \int_{0}^{\infty} \frac{1}{2 \sqrt{\pi a}} e^{-(\rho-R)^{2} / 4 a} \mathbf{T}(\rho) H(R-\rho) d \rho \\
& =\mathbf{T}(R) \lim _{a \rightarrow 0} \int_{0}^{\infty} \frac{1}{2 \sqrt{\pi a}} e^{-(\rho-R)^{2} / 4 a} H(R-\rho) d \rho \\
& =\mathbf{T}(R) \lim _{a \rightarrow 0} \int_{0}^{R} \frac{1}{2 \sqrt{\pi a}} e^{-(\rho-R)^{2} / 4 a} d \rho
\end{aligned}
$$

Taking into account the parity of $e^{-\rho^{2} / 4 a}$ produces

$$
\lim _{a \rightarrow 0} \int_{0}^{R} \frac{1}{2 \sqrt{\pi a}} e^{-(\rho-R)^{2} / 4 a} d \rho=\frac{1}{2} \lim _{a \rightarrow 0} \int_{0}^{\infty} \frac{1}{2 \sqrt{\pi a}} e^{-(\rho-R)^{2} / 4 a} d \rho=\frac{1}{2}
$$

and finally

$$
\left\langle(\mathbf{n} \delta)_{\Gamma} \theta_{r}, \varphi\right\rangle=\frac{1}{2}\left\langle(\mathbf{n} \delta)_{\Gamma} \theta, \varphi\right\rangle
$$

\section{Appendix B. Obsonov analytical solution for square voids in a periodic setting}

Obnosov [37] solved the problem of a rectangular inclusion located at the center of a periodic rectangular cell. For the comparison purpose, we consider the case where both the void and the cell are squared and the size of the former is half the size of the latter. In this case, the effective conductivity of the material is

$$
k_{e}=k_{r} / \sqrt{3}
$$

The Obnosov solution is based on a method using complex variables that produces, for a given overall flux of 1 along $x_{1}$, the heat flux inside the matrix represented by the complex flux $j^{c}$ defined by

$$
j^{c}=j_{1}-i j_{2}
$$


at each point of the complex plane identified by its complex affix $z=x_{1}+i x_{2} . j^{c}$ is given by:

$$
j^{c}=\theta J_{1}\left[e^{-i \pi / 6} \chi+e^{i \pi / 6} \chi^{-1}\right]
$$

where $J_{1}$ is the macroscopic flux. The two terms $\chi$ and $\theta$ are:

$$
\chi=\left[\frac{e^{i \pi / 4} \mathrm{dn}^{2}(2 K z \mid 1 / 2)-1 / \sqrt{2}}{\operatorname{dn}^{2}(2 K z \mid 1 / 2)-1 / \sqrt{2} e^{i \pi / 4}}\right]^{1 / 3}
$$

and

$$
\theta=\theta_{1}=\frac{\Gamma(5 / 6) \Gamma(2 / 3)}{\Gamma(3 / 4)^{2}}
$$

where $\operatorname{dn}(z \mid m)$ is the Jacobian elliptic function and $\Gamma$ is the Gamma function. $K$ is the value of the complete elliptic integral $K(m)$ at $m=0.5$, so $K=K(0.5)=1.854074677301372$ (for the special functions $K, \Gamma$ and dn, see [38]).

\section{Appendix C. Reference solution for circular holes}

The reference solution for the homogeneous conduction properties of a medium containing a periodic array of circular holes is based on a solution originally provided by Lord Rayleigh for composites containing circular inclusions [36]. This solution uses the expansion of the local temperature field into cylindrical harmonics, which can be written within the matrix, having taken into account the symmetries and the boundary conditions at the circular voids of radius $a$ and periodicity 1 along both directions:

$$
T=\sum_{n=1}^{\infty} \frac{B_{2 n-1}}{a^{2 n-1}}\left[\left(\frac{r}{a}\right)^{2 n-1}+\left(\frac{a}{r}\right)^{2 n-1}\right] \cos (2 n-1) \theta
$$

In Rayleigh's method, taking into account the interaction between all inclusions of the periodic setting produces an infinite system of linear equations for the coefficients $B_{2 n-1}$, which is written by using the lattice sums:

$$
S_{l}=\sum_{j=1}^{\infty}\left(x_{j}+i y_{j}\right)^{-l}
$$

where $x_{j}, y_{j}$ are the coordinates of the centers of the circles within the periodic lattice.

For a squared lattice, this sum is always real and is null except when $l=2$ or $l=4 p$. Denoting $X_{n}=$ $B_{2 n-2} /(n-1)$ !, the values of $X_{n}$ are solutions of the linear system:

$$
[M][X]=[V]
$$

where the components of the symmetric matrix $[M]$ are given by:

$$
M_{i j}=(2 i+2 j-3) ! S_{2(i+j-1)}+\delta_{i j} \frac{(2 i-1) !(2 i-2) !}{a^{2+4(i-1)}}
$$

and $V_{i}=\delta_{i 1}$, where $\delta$ is the Kronecker symbol. Since Rayleigh's work, a lot has been aeffected on the computation of the lattice sums. The values of these lattice sums are fully described in [39]

$$
\begin{aligned}
& S_{2}=\pi \\
& S_{4}=\frac{\pi^{4} A^{4}}{60} \\
& S_{8}=\frac{\pi^{8} A^{8}}{8400} \\
& S_{4 p}=4\left[\zeta(4 p)\left(1+\frac{(-1)^{p}}{2^{2 p}}\right)+\frac{2 \cos (4 p \alpha)}{5^{2 p}}\right]
\end{aligned}
$$

where $A=1.18034060, \alpha=\arccos (2 / \sqrt{5})$ and $\zeta$ is the Riemann zeta function [38].

The values of $B_{2 n-1}$ were obtained from a $7 \times 7$ matrix $[M]$. These values have been verified by comparison with the ones obtained from a collocation method [40]. 


\section{References}

[1] V. Myroshnychenko, C. Brosseau. Finite-element method for calculation of the effective permittivity of random inhomogeneous media. Phys. Rev, E 71 (2005) 016701.

[2] J. Eischen, S. Torquato, Determining elastic behavior of composites by the boundary element method, J. Appl. Phys. 74 (1993) 159-170.

[3] W.E Brown Jr., Solid mixture permittivities. J. Chem. Phys 23 (1955) 1514-1517.

[4] H. Moulinec, P. Suquet, A fast numerical method for computing the linear and nonlinear mechanical properties of composites, C. R. Acad. Sci. Puris 318 (1994) 1417-1423.

[5] J. Michel, H. Moulinec, P. Suquet, Effective properties of composite materials with periodic microstructure: a computational apprcach. Comput. Method Appl. Mech. Engrg. 172 (1999) 109-143.

[6] D.J. Eyre, G.W. Milton, A fnst numerical scheme for computing the response of composites using grid refinement, Eur. Phys J. Appl. Phys. 6 (1999) $41-47$.

[7] V. Monchiet, G. Bonnet, A polarization-based fast numerical method for computing the effective conductivity of composites, Int. J. Heat Fluid Flow 23 (2013) 1256-1271.

[8] G.W. Milton, The Theory of Composites, Vol, 6, Cambridge University Press, 2002.

[9] J. Michel, H. Moulinec, P. Suquet, A computarional method based on augmented lagrangians and fast fourier transforms for composites with high contrist, Comput. Model. Eng. Sci. 1 (2000) $79-88$.

[10] S. Brisand, L. Dormieux. FFT-based methods for the mechanics of composites: A general variational framework. Comput. Mater. Sci, 49 (2010) 663-671.

[11] J. Michel, H. Moulinec, P. Suquet, A computational scheme for linear and non-linear composites with arbitmry phase contrast, Intemat. J. Numer. Methods Engrg. 52 (2001) 139-160.

[12] H. Moulinec, F. Silva, Comparison of three acceleraied FFT-based schemes for computing the mechanical response of composite matrials, Internat. J. Numer. Methods Engrg 97 (2014) 960-985.

[13] M. Schneider, D. Wicht, T. Bohlke. On polarization-based schemes for the FFT-based computational homogenization of inelastic materials, Comput Mech, 64 (2019) 1073-1095.

[14] M. Schneider, Convergence of FFI-based homogenization for strongly heterogeneous media, Math. Methods AppL. Sci. 38 (2015) $2761-2778$.

[15] C. Bellis, H. Mculinec. P. Suquet, Eigendecomposition-based convergenee analysis of the Neumann series for laminated composites and discretization error estimation, Internat. J. Numer. Methods. Engrg. 121 (2020) 201-232.

[16] J. Vondrejc. J. Zeman, I. Marek, An FFT-based Gakerkin method for bomogenization of periodic media, Comput. Math. Appl. 68 (2014) $156-173$.

[17] S. Brisard, L. Domieux, Combining Galerkin approximntion techniques with the principle of Hashin and Shtrikman to derive a new FFT-based numerical method for the homogenization of composits, Comput. Methods Appl. Mech. Engrg. $217-220$ (2012) 197-212.

[18] J. Zeman, J. Vondreje, J. Novak, I. Marek, Accelerating a FFT-based solver for numerical homogenization of periodic media by conjugate gradients, J. Comput. Phys 229 (2010) 8065-8071.

[19] M. Schneider, On the Burzilai-Borwein basic scheme in FFT-based computational homogenization, Internat. J. Numer. Methods Engrg. 118 (2019) $482-494$

[20] D. Wicht, M. Schneider. T. Bohlke, On quasi-Newton methods in fast fourier transform-based micromechanics, Internat. J. Numer. Methods Engrg. (2019) http://dx.doi.org/10.1002/nme 6283.

[21] M. Kabel, T. Bchlke, M. Schneider, Efficient fixed point and Newton Krylov solvers for FFT- based homogenization of elasticity at large deformations; Comput. Mech. 54 (2014) 1497-1514.

[22] N, Mishra, J. Vondrejc, J. Zeman, A compuntive study on low-memory iterative solvers for FFT-based homogenization of periodic media, J. Comput. Phys. 321 (2016) 151-168.

[23] F. Willot, Fourier-based schemes for computing the mechanical response of composites with accurate local fields, C. R. Mec. 343 (2015) 232-245.

[24] F. Willot, B. Abdallah, Y, Pellegrini, Fourier-based schemes with modified Green operator for computing the electrical response of heterogenecus media with accurate local fields, Intemat. J. Numer. Methods Engrg. 98 (2014) 518-533.

[25] W.H. Mulker, Mathematical versus experimental stress analysis of inhomogeneities in solids, J. Physique IV 6 (1996) C1.139-C1.148.

[26] C. Dorn, M. Schneider. Lippmann-Schwinger solvers for the explicit jump discretization for thermal computational homogenization problems, Intemat. I. Numer. Methods Engrg. 118 (2019) 631-653.

[27] M. Leuschner, F. Fritzen, Fourier-accelerated nodal solvers. (FANS) for homogenization problems, Comput. Mech. 62 (2018) $359-392$.

[28] H. Moulinec, P. Suquet, G,W. Milton, Convergence of iterative methods based on Neumann series for composite materials: Theory and practice. Intemat. J. Numer. Methods Engrg. 114 (10) (2018) 1103-1130.

[29] H.L Nguyen, Q.D. To, Conductivity of composites with multipk polygonal aggregates, theoretical estimates and numerical solutions from polarization series, Internat, J. Engry. Sci. 123 (2018) 109-116.

[30] J. Wuttke, Form factor (Fourier shape transform) of polygon and polyhedron, 2017, arXiv preprint arXiv: 1703.00255.

[31] G. Bonnet, Effective properties of elastic periodic composite media with fibers, J. Mech. Phys Solids 55 (2007) $881-899$.

[32] S. Nemat-Nasser, T. Iwakumi, M. Hejazi, On compcsites with periodic structure, Mech. Mater. 1 (1982) 239-267.

[33] E. Krobner. Bounds for effective elastic moduli of disondered materials, J. Mech. Phys. Solids 25 (1977) $137-155$.

[34] S. Brisand, Reconstructing displacements from the solution to the periodic Lippmann-Sclawinger equation discretized on a uniform grid, Intemat. J. Numer. Methods Engrg. 109 (4) (2017) 459486.

[35] LC. Wrobel, The Boundary Element Method, Wiley, 2002 